\title{
Probing nonlinear velocity profiles of shear-thinning, nematic platelet dispersions in Couette flow using $x$-ray photon correlation spectroscopy
}

\author{
Y. Chen (陈一昊), ${ }^{1}$ O. Korculanin, ${ }^{2,}{ }^{3}$ S. Narayanan, ${ }^{4}$ J. Buitenhuis, ${ }^{2}$ S. A. Rogers, ${ }^{5}$ R. L. Leheny, ${ }^{1, \text { a) }}$ and M. P. \\ Lettinga ${ }^{2,3, b)}$ \\ 1) Department of Physics and Astronomy, Johns Hopkins University, Baltimore, Maryland 21218, \\ USA \\ 2) IBI-4, Forschungszentrum Jülich GmbH, 52428 Jülich, Germany \\ 3) Laboratory for Soft Matter and Biophysics, KU Leuven, B-3001 Leuven, Belgium \\ ${ }^{4)} X$-Ray Science Division, Argonne National Laboratory, Argonne, Illinois 60439, USA \\ ${ }^{5)}$ Department of Chemical and Biomolecular Engineering, University of Illinois Urbana-Champaign, Champaign, Illinois 61801,
} USA

(Dated: 5 July 2021)

We report experiments employing x-ray photon correlation spectroscopy (XPCS) to characterize the velocity profiles of complex fluids in Couette flow. The approach involves modeling the XPCS correlation functions obtained with the incident X-ray beam passing tangentially through the Couette cell gap at various distances from the inner wall. We first demonstrate the technique with measurements on a dilute colloidal dispersion in the Newtonian liquid glycerol, where the expected linear velocity profiles are recovered. We then employ the technique to map the shear-rate-dependent velocity profiles of a shear-thinning dispersion of nematically ordered Gibbsite platelets. The nonlinear velocity profiles of the Gibbsite dispersion include a narrow slip region adjacent to the outer wall and a band with small velocity gradient in the interior of the gap that evolves into a region increasingly resembling plug flow with increasing shear rate. Variations in the velocity profile along the vorticity direction indicate an instability in the interface between this region of small velocity gradient and a region of high velocity gradient near the inner wall. Analysis of the small-angle scattering patterns provides information about the spatial and temporal variations in the nematic order of the Gibbsite dispersion and their coupling to the velocity profile. Additional potential applications of this XPCS-based technique and comparisons with established methods for characterizing velocity profiles are discussed.

\section{INTRODUCTION}

The non-Newtonian flow behavior of a complex fluid implies that its viscosity is shear-rate dependent and that its velocity profile displays deviations from the linear flow. At low Reynolds numbers, non-Newtonian behavior is typically due to shear-induced changes in microstructure. Hence, an intimate connection exists between structural changes and velocity profile, which often varies in time as well as in space. Therefore, a complete understanding of complex, nonNewtonian flow requires the simultaneous acquisition of the mechanical response, the local velocity, and the local structure. In situ velocity profiling can be achieved by optical techniques like laser Doppler heterodyne light scattering ${ }^{1-3}$, optical coherence tomography $(\mathrm{OCT})^{4}$, and particle imaging velocimetry $^{5,6}$; however, these techniques require an optically transparent sample, although less so for OCT, which limits the range of applications. Rheo-NMR ${ }^{7-9}$ and ultrasonic rheovelocimetry (USV) ${ }^{10-13}$ have both been successfully applied to opaque samples, but these techniques also have important drawbacks. USV needs seeding with particles that scatter sound, and moreover, this technique does not supply any structural information. Rheo-NMR does supply both structural and flow information, but its drawbacks include long acquisition times, which preclude the study of temporal fluctuations. Here, we introduce a method that employs coherent

\footnotetext{
a) leheny@jhu.edu

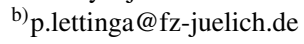

$\mathrm{X}$-ray scattering to measure the velocity profile in complex fluids under steady shear in Couette geometry and characterize their shear-dependent, heterogeneous microstructure simultaneously.

Coherent x-ray scattering, specifically x-ray photon correlation spectroscopy (XPCS), can be a sensitive probe of flow velocities due to the relative Doppler shift between scatterers in the flow moving at different velocities ${ }^{14,15}$. Combined with the spatial resolution afforded by the small beam size employed in coherent $\mathrm{x}$-ray scattering, this feature makes the technique a promising approach for characterizing nonlinear flow profiles in complex fluids. Several previous studies have explored the application of XPCS for characterizing flow velocities. Most of this work has focused on dilute colloidal dispersions, whose Newtonian flow properties served to demonstrate the potential of the technique for characterizing flow in microfluidic channels ${ }^{15-18}$ or under simple shear ${ }^{14,19,20}$. In particular, Urbani and coworkers ${ }^{17}$ exploited the micrometerscale beam size in XPCS to obtain spatially resolved information regarding Newtonian flow around corners and near constrictions in microfluidic channels. XPCS has also been combined with in situ rheometry to connect microstructural dynamics with rheological behavior in complex fluids. In one such application of "rheo-XPCS", Westermeier et al. ${ }^{20}$ characterized the transient structural dynamics in a colloidal fluid upon the cessation of shear at high Péclet number and correlated the behavior with the relaxation of shear-induced changes to the microstructure. In another, Chen et al. ${ }^{21}$ investigated the slow structural dynamics associated with stress relaxation in a colloidal glass following step strains and cor- 
related the decay in the stress with slow, flow-like dynamics in the glass.

In the present study, we employ XPCS to investigate the nonlinear flow behavior of dispersions of nanometer-scale colloidal platelets in the nematic phase under steady shear. Inorganic colloidal platelets in the nematic phase are ideal for illustrating the capabilities of XPCS for characterizing velocity profiles for several reasons. Nematic systems in shear flow can display strong shear thinning 22 due to reorientational motion of the director ${ }^{23-26}$, resulting in the formation of banded structures as observed by polarization microscopy under shear for colloidal rods ${ }^{27}$ and platelets ${ }^{28,29}$. In addition to the tumbling motion, confinement plays an important role for colloidal platelets, as the combination of wall anchoring and tumbling motion are predicted to cause flow instabilities $24,25,30-33$, and platelets are known to exhibit strong wall anchoring 34,35 '. Indeed, a strongly heterogeneous structure has been observed in pipe flow of colloidal platelet dispersions ${ }^{36}$. However, the relation between structure and flow still remains to be identified. Rheo-SAXS is a proven method to probe the coupling of complex flow behavior and the shear-induced changes in nematic structure ${ }^{37-39}$. However, information on the local flow velocity throughout the fluid is required for full understanding. As the relatively high opacity of the dispersions prohibits the use of optical techniques for velocity profiling, rheo-XPCS is the ideal method of choice.

In Sec. II below, we describe the fluids that are the subject of the study, which include the non-Newtonian dispersion of nematic platelets, composed of the mineral Gibbsite, and a second, Newtonian liquid composed of a dilute dispersion of spherical colloids in glycerol. In addition, we provide in Sec. II details regarding the experimental procedures in the rheo-XPCS measurements. Section III summarizes the principles in relating the results of an XPCS measurement on a fluid in Couette flow to its velocity profile. In Sec. IV A, we demonstrate the method using measurements on the Newtonian liquid, and in Sec. IV B we report the findings on the nematic Gibbsite dispersion, which include characterization of the shear-rate-dependent velocity profile and complementary results on the shear-rate-dependent, heterogeneous nematic order in the dispersion. Finally, in Sec. V we assess the capabilities of XPCS for characterizing velocity profiles and discuss possible future directions for applying the method to other complex fluids.

\section{EXPERIMENTAL METHODS}

\section{A. Fluids under study}

Two fluids were included in the experiments. The first was a dispersion of colloidal silica spheres dispersed in the Newtonian liquid glycerol at colloid volume fraction $1 \%$. The colloids had an average diameter of $D=182 \mathrm{~nm}$ with a polydispersity $\Delta D / D=1.5 \%$, as determined by SAXS from a highly dilute dispersion. Details on the synthesis of the colloids, which were employed in an earlier XPCS experiment ${ }^{40}$, can be found in Ref. ${ }^{41}$.

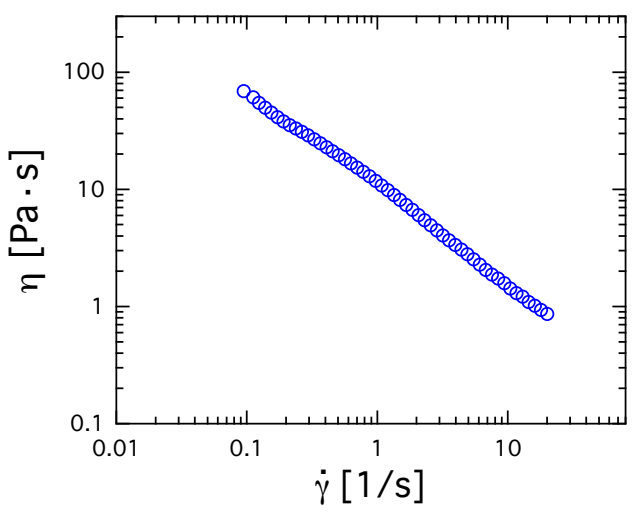

FIG. 1. Viscosity as a function of shear rate of the Gibbsite dispersion measured in a plate-plate geometry.

The other fluid was a dispersion of Gibbsite $\left[\gamma-\mathrm{Al}(\mathrm{OH})_{3}\right]$, which are charged, disk-like colloids with radius $125 \pm 16$ $\mathrm{nm}$ and thickness $8.4 \pm 2.8 \mathrm{~nm}$, in an $80 / 20$ glycerol/water solution with $12 \%$ colloidal volume fraction. The Gibbsite platelets were synthesized following a procedure from Ref. ${ }^{42}$ and characterized using transmission electron microscopy. At $12 \%$ volume fraction, the platelets are in a nematic phase in which the platelet faces orient in a common direction, which defines the local nematic director $\hat{n}^{42-44}$.

In the Couette cell, the platelets align with their faces parallel to the cell walls; that is, $\hat{n}$ is in the radial or gradient $(\nabla v)$ direction. Figure 1 shows the flow curve of the Gibbsite dispersion measured under constant shear rate in a plate-plate geometry. The behavior is typical of a shear-thinning material and similar to the behavior observed with other colloidal platelets $^{22,45}$. The shear stress $\sigma$ scales with the imposed shear rate in a sub-linear power-law relation, $\sigma \propto \dot{\gamma}^{m}$, with $m \approx 0.17$, so that $\eta \propto \dot{\gamma}^{-0.83}$.

\section{B. Rheo-XPCS}

The rheo-XPCS experiments were carried out at Sector 8ID of the Advanced Photon Source. The samples were contained in the Couette cell of a stress-controlled rheometer (Anton Paar MCR-301) mounted on the beam line. The Couette cell was composed of thin-walled polycarbonate allowing transmission x-ray scattering measurements with little attenuation or parasitic scattering from the container, as described previously ${ }^{21}$. The cell's inner and outer radii were nominally $R_{\text {in }}=5.5 \mathrm{~mm}$ and $R_{\text {out }}=6.1 \mathrm{~mm}$, respectively, leading to a nominal gap of $H=600 \mu \mathrm{m}$. A partially coherent, $10.9 \mathrm{keV}$ x-ray beam (wavelength $\lambda=0.114 \mathrm{~nm}$ ) of size $100 \times 20 \mu \mathrm{m}^{2}$ $(\mathrm{V} \times \mathrm{H})$ was focused vertically to a $3 \times 20 \mu \mathrm{m}^{2}$ spot on the sample. An area detector (X-spectrum LAMBDA 750K) ${ }^{46,47}$ positioned $4.91 \mathrm{~m}$ after the sample measured the scattering intensity over the range $0.02 \mathrm{~nm}^{-1}<|\mathbf{q}|<0.65 \mathrm{~nm}^{-1}$, where of $|\mathbf{q}|=4 \pi \sin (\theta / 2) / \lambda$ is the magnitude of the scattering wave vector at scattering angle $\theta$.

Measurements, depicted schematically in Fig. 2, were per- 


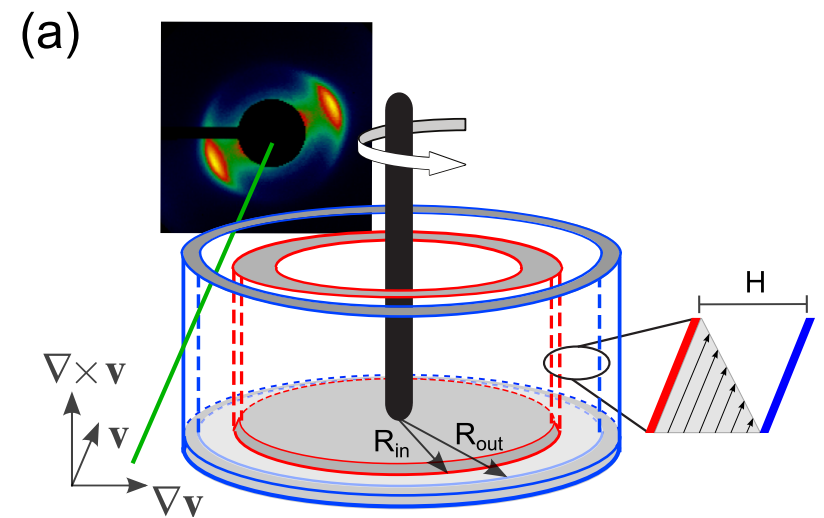

(b)

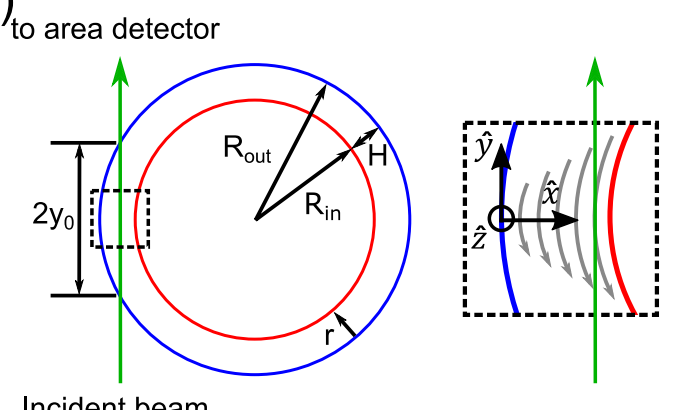

Incident beam

FIG. 2. Schematics depicting (a) a three-dimensional rendering and (b) a top view of the Couette cell with the incident coherent beam passing through the left edge of the cell. Points in the cell gap are specified by the distance from the outer wall $r$ and by the Cartesian coordinate system shown in (b), and the beam position is specified by the largest horizontal distance $x$ that it obtains from the outer wall when passing through the sample. An x-ray area detector behind the cell captures the coherent scattering intensity in transmission. Note that the distance to the detector and the Couette cell radii and gap width are not shown to scale.

formed with the axis of the Couette cell oriented vertically and the horizontal incident beam directed tangentially through the side of the cell at various distances from the outer wall. In this configuration, the incident beam, and hence the incident $\mathrm{x}$-ray wave vector $\mathbf{k}_{\mathbf{i}}$, was approximately parallel to the flow (v) direction, but due to the curvature of the Couette cell, it also made a small angle with the flow direction in the flowgradient $(v-\nabla v)$ plane that varied as it traversed the sample. Further, in the small-angle scattering regime of the measurements, $\mathbf{k}_{\mathbf{i}}$ and final x-ray wave vector $\mathbf{k}_{\mathbf{f}}$ were nearly parallel, so (since $\left|\mathbf{k}_{\mathbf{f}}\right| \approx\left|\mathbf{k}_{\mathbf{i}}\right|$ ) the scattering wave vector, $\mathbf{q}=\mathbf{k}_{\mathbf{f}}-\mathbf{k}_{\mathbf{i}}$, was essentially perpendicular to $\mathbf{k}_{\mathbf{i}}$. Therefore, the vertical $(\hat{z})$ component of $\mathbf{q}$ was in the vorticity $(\nabla \times v)$ direction, while the horizontal $(\hat{x})$ component was approximately parallel to the gradient direction but also had a small contribution parallel to the flow direction that again varied with position along the path of the incident beam. As described below, this small component of $\mathbf{q}$ parallel to the flow direction was important since it enables the characterization of the velocity profiles across the cell gap.

To measure a velocity profile, i.e., the velocity as a function of position in the gap of a fluid under steady shear, a constant shear rate was applied by the rheometer, and the measurements were taken once the sample reached a steady state. Shear rates applied to the dilute silica dispersion ranged from 0.0001 to $0.16 \mathrm{~s}^{-1}$, while three shear rates with nominal values $0.04,0.16$, and $0.32 \mathrm{~s}^{-1}$ (precise values given below) were applied to the Gibbsite dispersion. A series of measurements were made with the incident beam at eleven positions across the gap from the outer to the inner wall during steady shear at each rate. We characterize the position of the beam in each measurement by its maximum distance $x$ from the outer wall during its passage through the Couette cell, as depicted in Fig. 2(b).

At each beam position, coherent $\mathrm{x}$-ray scattering patterns were captured by the detector at a rate of $1000 \mathrm{fps}$ for $20 \mathrm{sec}-$ onds. Five such measurements were performed at each horizontal position, with the beam translated in the $z$-direction typically by $60 \mu \mathrm{m}$ between each measurement. The temporal variations in coherent scattering during each measurement caused by the dynamics of the fluid were characterized using the intensity autocorrelation function,

$$
g_{2}(\mathbf{q}, \tau)=\frac{<I(\mathbf{q}, t) I(\mathbf{q}, t+\tau)>_{t}}{<I(\mathbf{q}, t)>_{t}^{2}},
$$

where $I(\mathbf{q}, t)$ is the scattering intensity at scattering wave vector $\mathbf{q}$ and time $t, \tau$ is the delay time, and the brackets refer to averages over the duration of each measurement and over detector pixels within a small range of $\mathbf{q}$. The calculation of $g_{2}(\mathbf{q}, \tau)$ further involved employing the multi-tau correlation routine in which progressively larger sets of sequential images were first averaged when correlating across successively larger delay times so that the resulting delay times were spaced quasi-logarithmically at large delay time ${ }^{48}$. This multi-tau routing facilitates determining $g_{2}(\mathbf{q}, \tau)$ over a broad dynamic range and improves statistics, which proved important in the present study.

In the case of homodyne scattering, like in the present experiments, the intensity autocorrelation function is related to the field correlation function $g_{1}(\mathbf{q}, t)$ through the Siegert relation,

$$
g_{2}(\mathbf{q}, t)=1+\beta\left|g_{1}(\mathbf{q}, t)\right|^{2}
$$

where $\beta$ is the instrumental optical contrast, which varies from 0 to 1 depending on the coherence properties of the beam, the scattering geometry, and the solid angle subtended by a detector pixel. The field correlation function, which is equivalent to the intermediate scattering function, provides fundamental information about the dynamics in the sample. Specifically, for a system of $N_{p}$ scatterers, 


$$
g_{1}(\mathbf{q}, \tau)=\frac{\left\langle E^{*}(\mathbf{q}, \tau) E(\mathbf{q}, 0)\right\rangle}{\langle I(\mathbf{q})\rangle} \sim \sum_{k=1}^{N_{p}} \sum_{l=1}^{N_{p}}\left\langle b_{k}^{*}(\mathbf{q}, 0) b_{l}(\mathbf{q}, \tau) \exp \left[-i \mathbf{q} \cdot\left(\mathbf{r}_{k}(0)-\mathbf{r}_{l}(\tau)\right)\right]\right\rangle
$$

where $b_{k}(\mathbf{q}, \tau)$ is the scattering length of the $k^{t h}$ scatterer, $\mathbf{r}_{k}(\tau)$ is the scatterer's position, $E(\mathbf{q}, \tau)$ is the electric field associated with the scattered wave, which is related to the scattering intensity by $I(\mathbf{q}, \tau)=E^{*}(\mathbf{q}, \tau) E(\mathbf{q}, \tau)$, and the brackets indicate an ensemble average.

\section{XPCS UNDER COUETTE FLOW}

In a homodyne scattering measurement, the decay of the autocorrelation function for a colloidal dispersion under flow generally has three contributions ${ }^{14,49}$. One results from gradients in the flow velocity across the illuminated volume, a second is from dynamics intrinsic to the dispersion like diffusion of the colloids, and a third results from the transit of fluid into and out of the illuminated volume. Under appropriate conditions, these contributions can be factorized ${ }^{14,49}$, so that the theoretical form of the normalized autocorrelation function, $\Delta g_{2}=\left(g_{2}-1\right) / \beta$, can be expressed as

$$
\Delta g_{2}(\mathbf{q}, \tau)=\Delta g_{2}^{d}(\mathbf{q}, \tau) \Delta g_{2}^{\omega}(\mathbf{q}, \tau) \Delta g_{2}^{t}(\mathbf{q}, \tau)
$$

where $\Delta g_{2}^{d}, \Delta g_{2}^{\omega}$, and $\Delta g_{2}^{t}$ represent the contributions from diffusion, flow gradient, and transit, respectively.

For the conditions of our measurements, in which the flow is nearly parallel to the incident beam, the decorrelation time due to transit is much larger than those due to the other contributions. Therefore, we can make the approximation $\Delta g_{2}^{t}(\mathbf{q}, \tau)=1$. The diffusion term typically takes an exponential form,

$$
\Delta g_{2}^{d}(\mathbf{q}, \tau)=e^{-\Gamma_{D} \tau}
$$

where $\Gamma_{D}(\mathbf{q})$ is the diffusive decay rate at wave vector $\mathbf{q}$. The flow gradient term for a system of identical scatterers under flow can be evaluated by

$$
\Delta g_{2}^{\omega}(\mathbf{q}, \tau)=\frac{1}{N_{p}^{2}} \sum_{k=1}^{N_{p}} \sum_{l=1}^{N_{p}} \cos \left[\mathbf{q} \cdot\left(\mathbf{v}_{k}-\mathbf{v}_{l}\right) \tau\right]
$$

where $N_{p}$ again is the number of scatterers in the illuminated region, and $\mathbf{v}_{k}$ is the velocity due to flow of the $k^{\text {th }}$ scatterer. Considering the scattering geometry shown in Fig. 2, we see that in the small-angle scattering regime $\mathbf{q}$ is in the $x-z$ plane, while $\mathbf{v}$ is in the $x-y$ plane; hence, $\mathbf{q} \cdot \mathbf{v}=q_{x} v_{x}$. Replacing the sums over the scatterers in Eq. (6) with integrals over the incident beam path, we therefore obtain

$$
\begin{aligned}
\Delta g_{2}^{\omega}(\mathbf{q}, \tau ; x)= & \frac{1}{4 y_{0}^{2}} \int_{-y_{0}}^{y_{0}} \int_{-y_{0}}^{y_{0}} \cos \left\{q_{x} \tau\left[v_{x}\left(x, y_{k}\right)-v_{x}\left(x, y_{l}\right)\right]\right\} d y_{k} d y_{l} \\
= & \frac{1}{4 y_{0}^{2}} \int_{-y_{0}}^{y_{0}} \int_{-y_{0}}^{y_{0}}\left\{\cos \left[q_{x} \tau v_{x}\left(x, y_{k}\right)\right] \cos \left[q_{x} \tau v_{x}\left(x, y_{l}\right)\right]\right. \\
& \left.\quad+\sin \left[q_{x} \tau v_{x}\left(x, y_{k}\right)\right] \sin \left[q_{x} \tau v_{x}\left(x, y_{l}\right)\right]\right\} d y_{k} d y_{l}
\end{aligned}
$$

where $y_{0}=\sqrt{R_{\text {out }}^{2}-\left(R_{\text {out }}-x\right)^{2}}$ is half the path length travelled by the beam through the fluid at a given beam position $x$, as depicted in Fig. 2(b). The circular stream lines in Couette flow imply $v_{x}(x, y)$ is an odd function of $y$, hence the integral over the sine terms is zero, leading to

$$
\Delta g_{2}^{\omega}(\mathbf{q}, \tau ; x)=\frac{1}{y_{0}^{2}}\left[\int_{0}^{y_{0}} \cos \left[q_{x} \tau v_{x}(x, y)\right] d y\right]^{2} .
$$

Together with the contribution from diffusion, the full form of $\Delta g_{2}(\mathbf{q}, \tau ; x)$ for scattering from a fluid under flow in the geometry of Fig. 2 therefore is

$$
\Delta g_{2}(\mathbf{q}, \tau ; x)=\frac{e^{-\Gamma_{D} \tau}}{y_{0}^{2}}\left[\int_{0}^{y_{0}} \cos \left[q_{x} \tau v_{x}(x, y)\right] d y\right]^{2} .
$$

In Sec. IV A below, we adapt Eq. (9) to the case of linear shear flow and employ it to analyze measurements on the dilute dis- persion of silica colloids in glycerol. Those results provide a framework for analyzing the measurements on the Gibbsite dispersion, for which nonlinear velocity profiles are obtained, as described in Sec. IV B.

\section{RESULTS}

\section{A. Newtonian fluid: linear velocity profile}

Figure 3 shows a set of correlation functions measured on the dilute silica colloid dispersion in glycerol, whose rheology is that of a Newtonian fluid. The correlation functions were measured at $q_{x}=0.032 \mathrm{~nm}^{-1}$ and $q_{z}=0$ at nominal shear rates $0.0001,0.04$, and $0.16 \mathrm{~s}^{-1}$ with the incident beam at different positions $x$ relative to the outer wall. For clarity, only data 
from six positions are shown; data from all eleven positions can be found in the Supplementary Material. Each data set is the average over five measurements at the same $x$ but different vertical positions in the Couette cell.

As Fig. 3 shows, $\Delta g_{2}\left(q_{x}, \tau ; x\right)$ decays at smaller $\tau$ as the measurement position $x$ increases from the outer to the inner wall. We can understand this variation qualitatively as follows. As the incident beam passes through the Couette cell, the magnitude and direction of the local flow velocity varies with location $y$ along the beam path, where $y=0$ is taken as the midpoint of the path, as shown in Fig. 2(b). During the first half of the beam's path through the sample $(y<0)$, the velocity increases with $y$ since the beam traverses points that are increasingly far from the stationary outer wall. The velocity peaks at the midpoint of the beam path, and then decreases for the rest of the path in a symmetric manner. The magnitude of the angle between the local velocity and the beam varies in the opposite way; it first decreases with increasing $y$ when $y<0$ and increases when $y>0$. Therefore, when the measurement position is closer to the inner wall (larger $x$ ), the beam not only passes through higher velocity regions, but also makes larger angles with the local velocity in part of its path, so together $q_{x} v_{x}$ in Eq. (9) reaches larger values, leading to faster decay.

To model the correlation functions quantitatively we assume the glycerol dispersion has a uniform shear rate $\dot{\gamma}$ across the cell gap and hence a linear velocity profile. The $\hat{x}$ component of the velocity along the path of the incident beam at position $(x, y)$ in this case is

$$
v_{x}(x, y)=\dot{\gamma}\left[R_{\text {out }} \frac{y}{\sqrt{\left(R_{\text {out }}-x\right)^{2}+y^{2}}}-y\right] .
$$

Inserting this result into Eq. (9), we obtain an expression for $\Delta g_{2}\left(q_{x}, \tau ; x\right)$ for a linear profile,

$$
\Delta g_{2}\left(q_{x}, \tau ; x\right)=\frac{e^{-\Gamma_{D} \tau}}{y_{0}^{2}}\left[\int_{0}^{y_{0}} \cos \left\{q_{x} \dot{\gamma} \tau\left[R_{\mathrm{out}} \frac{y}{\sqrt{\left(R_{\text {out }}-x\right)^{2}+y^{2}}}-y\right]\right\} d y\right]^{2}
$$

To obtain the decay rate $\Gamma_{D}$ due to diffusion, we examine $\Delta g_{2}\left(q_{x}, \tau ; x\right)$ from the measurements at the smallest nominal shear rate, $\dot{\gamma}=0.0001 \mathrm{~s}^{-1}$. Note that the correlation functions at this shear rate (Fig. 3(a)) show less variation with $x$ than those at the higher shear rates (Figs. 3(b) and (c)). This smaller variation is a consequence of the significant contribution made by diffusion, which is independent of beam position, at the smallest shear rate. In particular, at the outermost beam position, where the local flow velocity is very small and largely parallel to the incident beam, we can make the approximation that the contribution from the flow gradients to the decay of $\Delta g_{2}\left(q_{x}, \tau ; x\right)$ is negligible $\left(\Delta g_{2}^{\omega}=1\right)$. The solid line in Fig. 3(a) shows the result of a fit of an exponential decay to this data set, which describes the data accurately and gives $\Gamma_{D}=0.74 \pm 0.04 \mathrm{~s}^{-1}$. This value is close to the expected decay rate for the spheres diffusing in glycerol.

Our analysis of $\Delta g_{2}\left(q_{x}, \tau ; x\right)$ focuses on the higher rates where the contribution from flow to the decay of the correlation function is more pronounced than in Fig. 3(a). This contribution is apparent in Figs. 3(b) and (c) not only from the variation in decay rate with beam position but from the fasterthan-exponential shape of the decay. In principle, with $\Gamma_{D}$ known, Eq. (11) provides an exact expression for $\Delta g_{2}\left(q_{x}, \tau\right)$ at beam position $x$ for a linear velocity profile with given Couette cell radius $R_{\text {out }}$ and shear rate $\dot{\gamma}$. However, while the relative values of $x$ for different measurements were precisely controlled by the motors that translated the rheometer in the beam between measurements, the absolute values of $x$ with respect to the outer wall could only be approximated at the time of the experiment. Therefore, in modeling $\Delta g_{2}\left(q_{x}, \tau ; x\right)$ at different $x$, we introduce a free parameter $x_{1}$ that specifies the position of the beam in the measurement closest to the outer wall, and the value of this parameter sets the positions of the other ten measurements across the gap. A second uncertainty in the measurements was the gap width of the Couette cell, which potentially differed from the nominal $600 \mu \mathrm{m}$ due to small deviations from specifications during manufacturing of the polycarbonate cell or deformation of the thin cell walls over time. To account for this uncertainty when modeling the data, we assume that the inner radius is $R_{\text {in }}=5.5$ mm, and we take the gap width $H$ as a second free parameter. Consequently, $R_{\text {out }}$ is taken as $R_{\text {out }}=R_{\text {in }}+H$, and the true shear rate is taken as $\dot{\gamma}=\dot{\gamma}_{n} H_{n} / H$, where $\dot{\gamma}_{n}$ and $H_{n}=600$ $\mu \mathrm{m}$ are the nominal shear rate set by the rheometer and nominal gap width, respectively. A least-squared minimization was adopted to fit the eleven $\Delta g_{2}\left(q_{x}, \tau ; x\right)$ at various $x$ and a given nominal shear rate simultaneously using Eq. (11) with $x_{1}$ and $H$ as free parameters. Results of the fits are depicted by solid lines in Figs. 3(b) and (c) for the data at nominal shear rates 0.04 and $0.16 \mathrm{~s}^{-1}$, respectively. (The results of the fits at all eleven beam positions at these shear rates can be found in the Supplementary Material.)

As Figs. 3(b) and (c) illustrate, the fits agree well with the measured correlation functions over the main parts of the decays of $\Delta g_{2}\left(q_{x}, \tau ; x\right)$, and importantly the model accurately captures the variation in the decay rate with the position of the beam in the gap. However, the model line shapes also include oscillations in the tails of the correlation functions at large $\tau$ that are only partially resolved in the experimental data. While such oscillations are a typical feature of correlation functions measured under flow ${ }^{15}$, we attribute their attenuation in the experimental results to the multi-tau routine employed to obtain $\Delta g_{2}\left(q_{x}, \tau ; x\right)$ from the x-ray images, which has the effect of smearing non-monotonic features in the correlation function at large delay times, and to the finite width of the beam, which similarly has the effect of smearing such features by 
effectively averaging over a range of $x$. Despite these discrepancies in the tails of $\Delta g_{2}\left(q_{x}, \tau ; x\right)$, the overall quality of the fits, which include only two parameters for all eleven correlation functions, indicates the validity of Eq. (11) and of our assumption of a linear velocity profile in the dilute colloid dispersion. The fit results yielded $x_{1}=33 \pm 2 \mu \mathrm{m}$ and $H=629$ $\pm 17 \mu \mathrm{m}$. This difference between the actual and nominal gap widths implies the actual applied shear rates were slightly smaller than the set values. Specifically, the actual shear rates of the measurements were $9.5 \times 10^{-5}, 0.038$, and $0.15 \mathrm{~s}^{-1}$ for the nominal rates of $0.0001,0.04$, and $0.16 \mathrm{~s}^{-1}$, respectively. Importantly, the same Couette cell was used in the measurements on the Gibbsite dispersion, and the reference point for the beam position in the cell was unchanged between experiments, hence these parameters were also employed in analyzing the Gibbsite results described below.

\section{B. Gibbsite dispersion: nonlinear velocity profile and slip region}

Figure 4(a) shows an example of an area detector image of the scattering pattern obtained from the Gibbsite dispersion under shear. This specific measurement was taken at $x=55$ $\mu \mathrm{m}$ while the dispersion was under shear at $0.038 \mathrm{~s}^{-1}$. The anisotropy in the scattering intensity is indicative of the nematic order in the dispersion, with the two lobes of scattering near $q=0.083 \mathrm{~nm}^{-1}$ corresponding to an interparticle structure factor peak related to the nearest-neighbor correlations between platelets along the direction $\hat{n}$ of the nematic director (i. e., normal to the face of the platelets). As illustrated in the cartoon in Fig. 4(b), the scattering pattern plotted in Fig. 4(a) indicates that the director $\hat{n}$ is in this case oriented approximately, but not precisely, parallel to $\hat{x}$. This means that the platelets align nominally with their faces parallel to the Couette cell walls, and hence $\hat{n}$ is approximately along the gradient direction. The deviation of $\hat{n}$ from this direction, expressed in terms of the angle $\theta_{m}$ between $\hat{n}$ and $\hat{x}$ is discussed below in Sec. IV B 4.

Figures 5(a), (b), and (c) display results for $\Delta g_{2}\left(q_{x}, \tau ; x\right)$ at $q_{x}=0.03 \mathrm{~nm}^{-1}$ and $q_{z}=0$ obtained from measurements on the Gibbsite dispersion at shear rates of $0.038,0.15$, and $0.31 \mathrm{~s}^{-1}$, respectively. Each set of data corresponds to measurements at the same eleven positions $x$ in the Couette cell gap as those in the measurements on the dilute colloid dispersion, and each curve is again an average over five measurements at the same $x$ but different vertical positions in the cell. Data from all eleven horizontal positions are shown in the Supplementary Material. A comparison of these autocorrelation functions with those obtained with the dilute colloid dispersion at the same shear rates immediately indicates a strongly nonlinear velocity profile in the Gibbsite dispersion. For instance, comparing the $\Delta g_{2}\left(q_{x}, \tau ; x\right)$ at a shear rate of $0.038 \mathrm{~s}^{-1}$ in Fig. 3(b) with those in 5(a), one sees that at the position closest to the outer wall, $x=33 \mu \mathrm{m}, \Delta g_{2}\left(q_{x}, \tau ; x\right)$ of the Gibbsite dispersion has a decay time that is almost five times faster than that of the dilute colloid dispersion. This difference is evidence for a large velocity gradient indicative of a high-shear, slip region

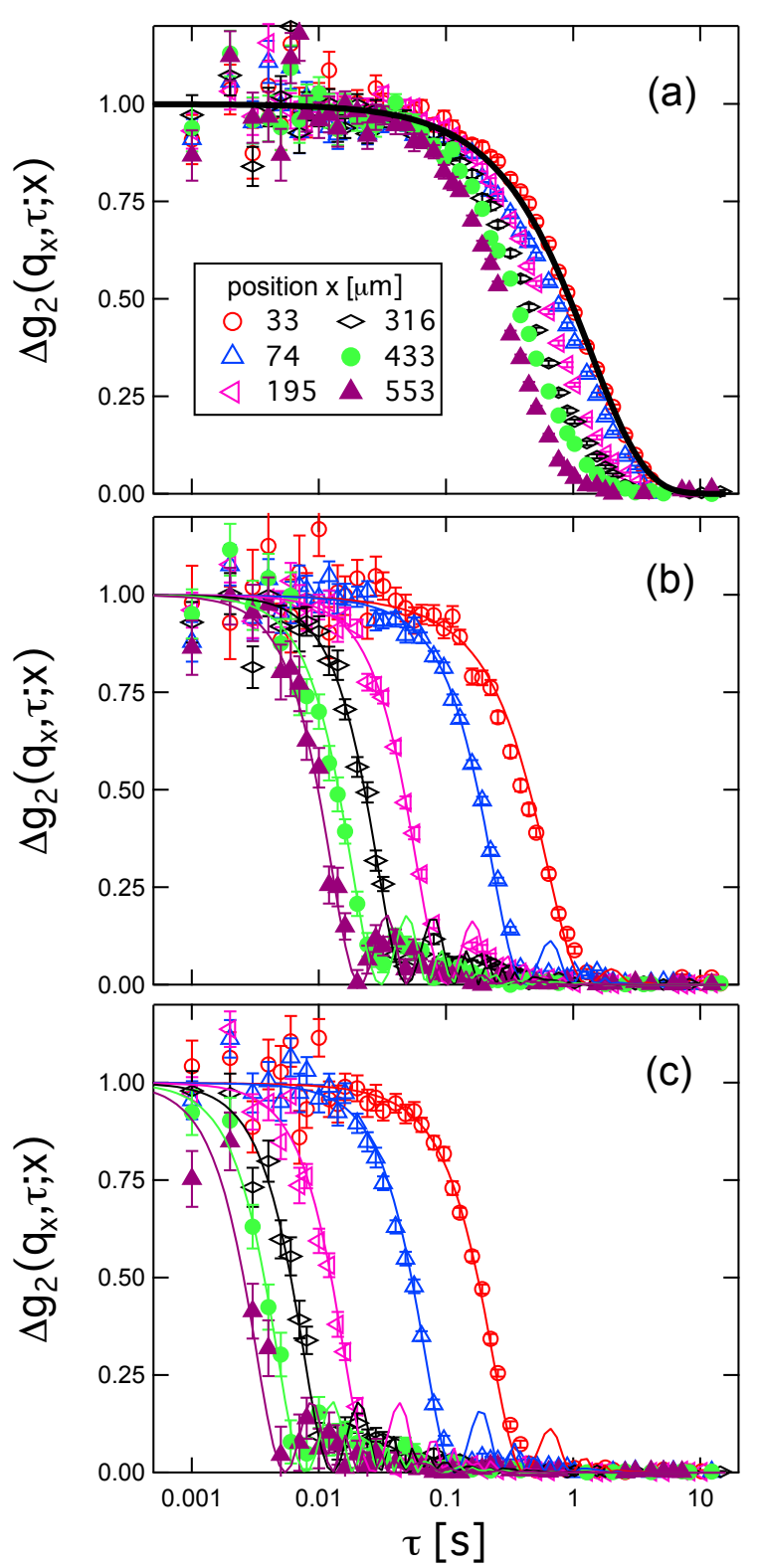

FIG. 3. $\Delta g_{2}\left(q_{x}, \tau ; x\right)$ measured on the silica colloid dispersion in glycerol at $q_{x}=0.032 \mathrm{~nm}^{-1}$ and $q_{z}=0$ under nominal shear rates (a) 0.0001 , (b) 0.04 , and (c) $0.16 \mathrm{~s}^{-1}$ at 6 different positions in the gap as specified by the incident beam's distance $x$ from outer wall listed in the legend. The thin solid lines in (b) and (c) show the results of fits using Eq. (11). The thick solid line in (a) shows the result of a fit to the data at $x=33 \mu \mathrm{m}$ using a simple exponential function. For clarity, only data from six positions are shown; data from all eleven positions can be found in Supplementary Material.

in the Gibbsite dispersion near the outer wall, which has been observed in many complex fluids ${ }^{50,51}$.

This slip region has a strong influence on the measurements at all $x$, since, as seen in Fig. 2, regardless of $x$ the incident beam passes through the region near the outer wall upon entering and exiting the fluid. Indeed, a measurement at a given $x$ depends on the flow dynamics not only at that 


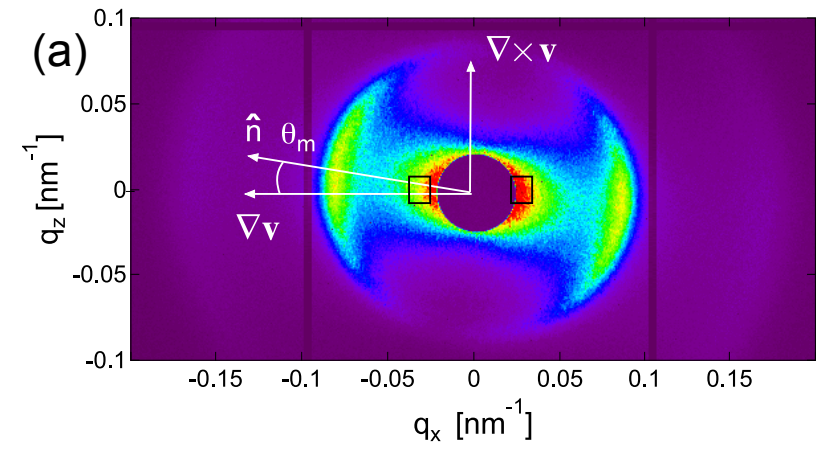

(b)

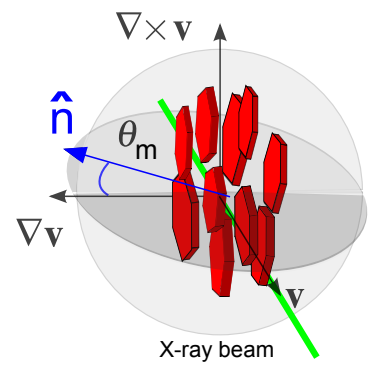

FIG. 4. (a) Scattering pattern from the Gibbsite dispersion under shear rate $0.038 \mathrm{~s}^{-1}$ with the incident beam passing tangentially through the side of the Couette cell at $x=55 \mu \mathrm{m}$ from outer wall. The two rectangular regions, centered on $q_{x}= \pm 0.03 \mathrm{~nm}^{-1}$ and $q_{z}=$ 0 and spanning $0.012 \times 0.015 \mathrm{~nm}^{-2}\left(q_{x} \times q_{z}\right)$, indicate the regions included in calculating $\Delta g_{2}\left(q_{x}, \tau ; x\right)$. (b) Cartoon of the nematic platelets oriented with respect to the shear flow.

distance from the outer wall but at all distances from 0 to $x$. Consequently, $\Delta g_{2}\left(q_{x}, \tau ; x\right)$ measured at different $x$ are not independent of each other. This interdependence combined with the clearly nonlinear flow in the Gibbsite dispersion creates a challenge for analyzing the $\Delta g_{2}\left(q_{x}, \tau ; x\right)$ at different $x$ to determine the velocity profile. In particular, while one can obtain exact expressions for $\Delta g_{2}\left(q_{x}, \tau ; x\right)$ from Eq. (9) for an arbitrary velocity profile, the experiments present the inverse problem of needing to obtain $v(x, y)$ from the measured $\Delta g_{2}\left(q_{x}, \tau ; x\right)$. Therefore, as described below, to analyze the results for the Gibbsite dispersion, we adopt model functional forms for $v(x, y)$ using two different approaches and test the suitability of each by employing them in fits to $\Delta g_{2}\left(q_{x}, \tau ; x\right)$. First, we approximate the velocity profile across the gap by a sequence of bands with different shear rates; that is, we model the nonlinear velocity profile as a piece-wise linear function. Second, we employ a power-law velocity profile that is known to lead to shear thinning behavior like that in Fig. 1. In addition, to obtain the contribution from diffusion to the correlation function line shape, we fit $\Delta g_{2}\left(q_{x}, \tau\right)$ measured on the Gibbsite dispersion under quiescent conditions (i.e, no applied shear). Results of this measurement at $q_{x}=0.03 \mathrm{~nm}^{-1}$ are shown by the black asterisks in Fig. 5(a), and the line through the data shows the result of a fit using an exponential form from which the diffusive decay rate $\Gamma_{D}=0.42 \pm 0.01 \mathrm{~s}^{-1}$ is obtained. (Note since this decay rate is measured at a wave vector along $\hat{x}$, it corresponds to the diffusion of the platelets parallel to $\hat{n}$. Measurements with the wave vector along $\hat{z}$ in-

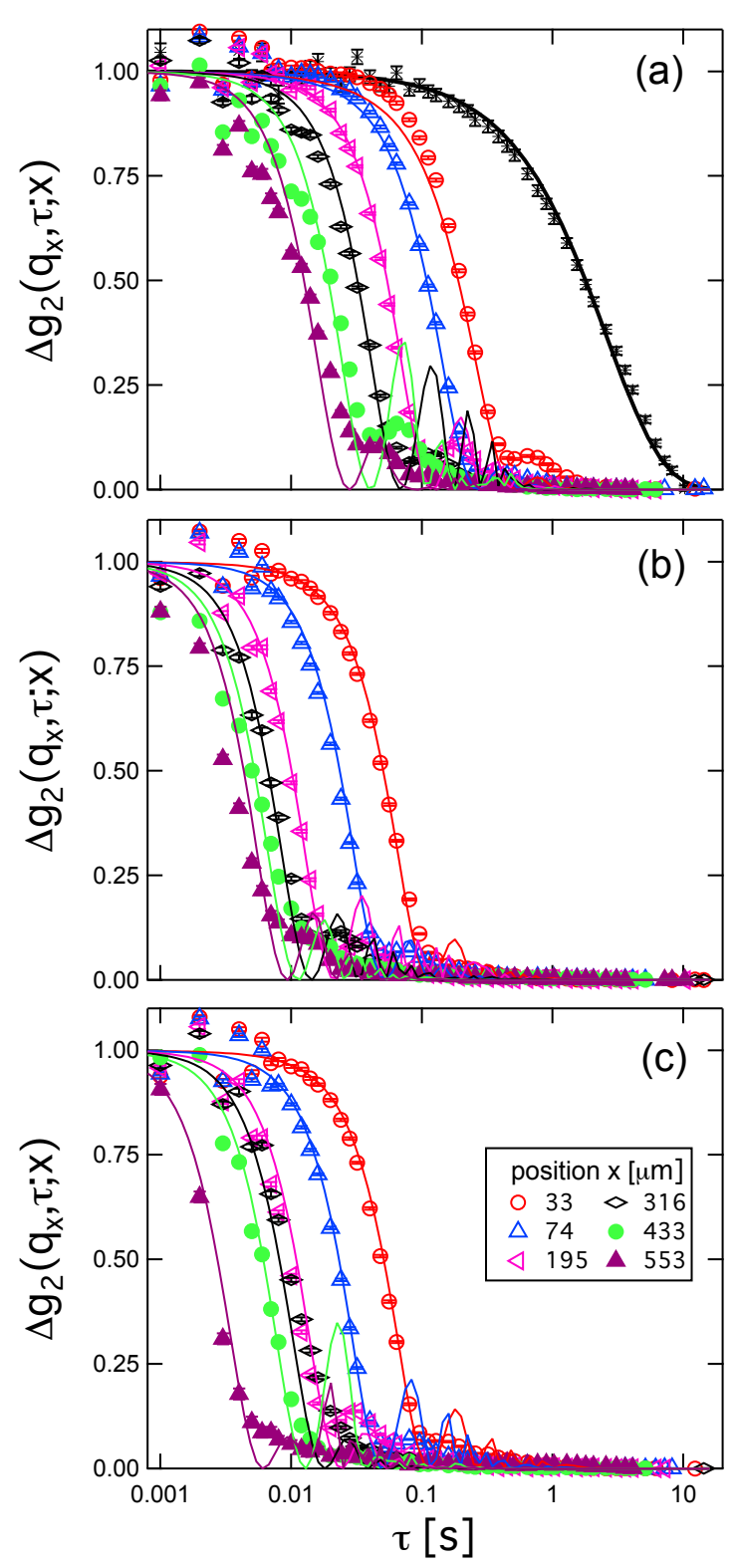

FIG. 5. $\Delta g_{2}\left(q_{x}, \tau ; x\right)$ measured on the Gibbsite dispersion at $q_{x}=0.03$ $\mathrm{nm}^{-1}$ and $q_{z}=0$ under constant shear rate (a) 0.038 , (b) 0.15 , and (c) $0.31 \mathrm{~s}^{-1}$, respectively, at 6 positions in the gap as specified by the distances from the outer wall listed in the legend. The thin solid lines show the results of fits using the piece-wise linear model (Eq. (14)) with five shear bands $(n=5)$. Also shown in (a) by the black asterisks is $\Delta g_{2}\left(q_{x}, \tau\right)$ measured on the dispersion under quiescent conditions at $q_{x}=0.03 \mathrm{~nm}^{-1}$. The thick solid line through the quiescent data shows the result of a fit using an exponential form to characterize diffusion parallel to the nematic director. For clarity, only data from six positions is shown; data from all eleven positions can be found in the Supplementary Material.

dicate the diffusion in the nematic dispersion is anisotropic, and the diffusion coefficient is much larger perpendicular to $\hat{n}$ than parallel.) 


\section{Piece-wise linear model}

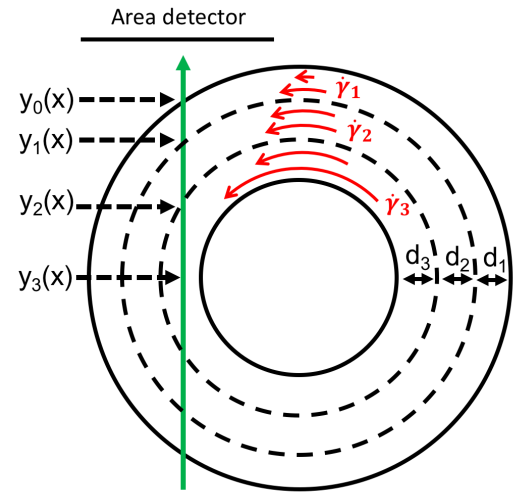

Incident beam

FIG. 6. Schematic of a top view of the Couette cell depicting a velocity profile in the piece-wise linear model comprised of three shear bands $(n=3)$ of width $d_{i}$ and shear rate $\dot{\gamma}_{i}$. At the beam position shown, the incident beam passes through all three shear bands.
In the piece-wise linear model, depicted schematically in Fig. 6, a nonlinear velocity profile is approximated by dividing the Couette cell gap into a set of $n$ concentric bands each with a different width $d_{i}$ and unique local shear rate $\dot{\gamma}_{i}$, where $i$ is indexed starting from the outer wall. The velocity profile is obtained by treating the $d_{i}$ and $\dot{\gamma}_{i}$ as free parameters that are optimized by fitting to all eleven $\Delta g_{2}\left(q_{x}, \tau ; x\right)$ simultaneously. (Note that since the widths of the bands must sum to the gap spacing, $\sum d_{i}=H$, the total number of free parameters for $n$ bands is $N=2 n-1$.) Within the $k^{\text {th }}$ shear band, the magnitude of the velocity at position $(x, y)$ is

$$
v_{k}(x, y)=\sum_{i=1}^{k} \dot{\gamma}_{i} d_{i}+\dot{\gamma}_{k}\left[R_{\text {out }}-\sqrt{y^{2}+\left(R_{\text {out }}-x\right)^{2}}-\sum_{i=1}^{k} d_{i}\right]
$$

and its $x$-component is

$$
v_{k, x}(x, y)=\sum_{i=1}^{k} \dot{\gamma}_{i} d_{i} \frac{y}{\sqrt{\left(R_{\text {out }}-x\right)^{2}+y^{2}}}+\dot{\gamma}_{k}\left(R_{\text {out }}-\sum_{i=1}^{k} d_{i}\right) \frac{y}{\sqrt{\left(R_{\text {out }}-x\right)^{2}+y^{2}}}-\dot{\gamma}_{k} y .
$$

The predicted form of $\Delta g_{2}\left(q_{x}, \tau ; x\right)$, including the contribution from diffusion, at beam position $x$ is thus

$$
\Delta g_{2}\left(q_{x}, \tau ; x\right)=\frac{e^{-\Gamma_{D} \tau}}{y_{0}^{2}}\left[\sum_{i=1}^{n^{\prime}} \int_{y_{i}(x)}^{y_{i-1}(x)} \cos \left[q_{x} v_{i, x}(x, y) \tau\right] d y\right]^{2}
$$

where $n^{\prime}$ is the number of shear bands that the incident beam at position $x$ passes through, and the limits of the integral are the positions in $y$ at which the incident beam crosses shear-band boundaries:

$$
y_{i}(x)= \begin{cases}\sqrt{R_{\text {out }}^{2}-\left(R_{\text {out }}-x\right)^{2}}, & i=0 \\ \sqrt{\left(R_{\text {out }}-\sum_{j=1}^{i} d_{j}\right)^{2}-\left(R_{\text {out }}-x\right)^{2},}, & i=1,2, \ldots, n^{\prime}-1 \\ 0, & i=n^{\prime} .\end{cases}
$$

Of course, the larger the number of bands $n$ that are included, the more finely a nonlinear velocity profile can be modeled but at the expense of an increasing number of free parameters. To determine the minimum number of bands that provides an adequate approximation of the velocity profile in the Gibbsite dispersion, we plot in Fig. 7 the mean dis- crepancy $\chi$, defined as the square root of averaged squared difference between the best fits using Eq. (14) and the data points of all eleven $\Delta g_{2}\left(q_{x}, \tau ; x\right)$ at each nominal shear rate, where in each case the eleven correlation functions are fit simultaneously, as a function of the number of fitting parameters. For reference, the horizontal lines in Fig. 7 indicate the 


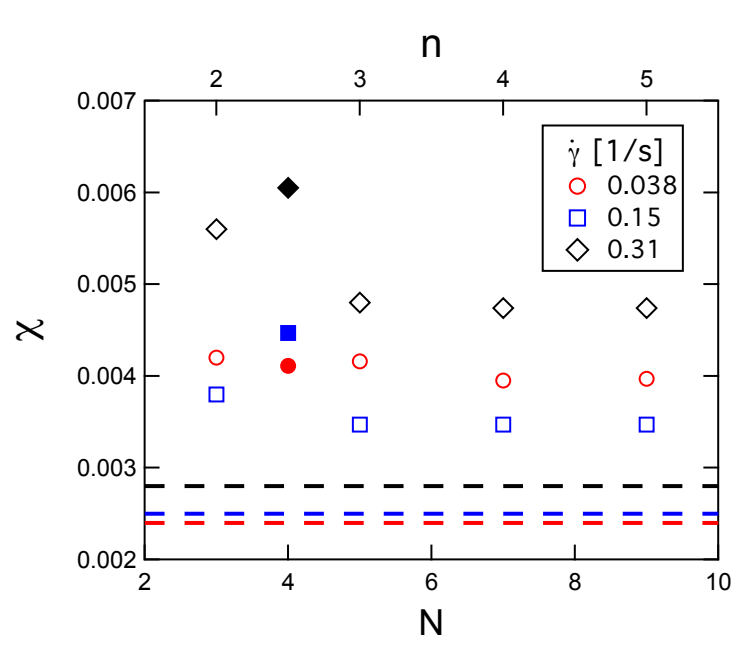

FIG. 7. The mean discrepancy $\chi$ of fits as a function of number of fitting parameters $N$. The open symbols are the mean discrepancies of fits using the piece-wise linear model with number $n$ of bands from two to five. The closed symbols are the discrepancies of fits using the power-law model, which has four fitting parameters. The dashed lines indicate discrepancies of fits in which each individual $\Delta g_{2}\left(q_{x}, \tau ; x\right)$ is fit using an empirical, compressed-exponential line shape.

mean discrepancies obtained from fitting each $\Delta g_{2}\left(q_{x}, \tau ; x\right)$ individually with an empirical compressed-exponential lineshape, which captures the shape of the correlation functions well and therefore can be regarded as providing an estimate of the contribution to the discrepancy from statistical noise. (Note such analysis employs $N=22$ fit parameters.) As the figure illustrates, the discrepancy decreases with an increasing number of bands up to $n=4$ but appears to plateau by $n=5$. The value of the discrepancy at $n=5$ is larger than the estimated contribution from statistical noise, indicating some persistent systematic deviations between the model and the data. The lines in Fig. 5 show the results of fits at each nominal shear rate using the piece-wise linear model with $n=5$. As the figure indicates, the model captures the measured correlations accurately in the outer portion of the gap $(\operatorname{smaller} x)$, but deviations are apparent close to the inner wall. One contributor to these deviations is heterogeneity in the velocity profiles along the vorticity direction that distort the experimental $\Delta g_{2}\left(q_{x}, \tau ; x\right)$, which averages over multiple measurements at different heights in the Couette cell. These heterogeneities are discussed in Sec. IV B 3 below. In addition, as with the results for the dilute silica dispersion, the model line shapes include oscillations in the tails of the correlations functions at large $\tau$ that are largely smeared out in the data. We attribute this smearing in the experimental results to the same effects described in Sec. IV A.

Figure 8 shows the velocity profiles of the Gibbsite dispersion under shear rates $0.038,0.15$, and $0.31 \mathrm{~s}^{-1}$ obtained from the best fits using Eq. (14) with $n=5$. Note that the velocity gradient in each band and the widths of the bands, which are free parameters that can differ at the different engineered
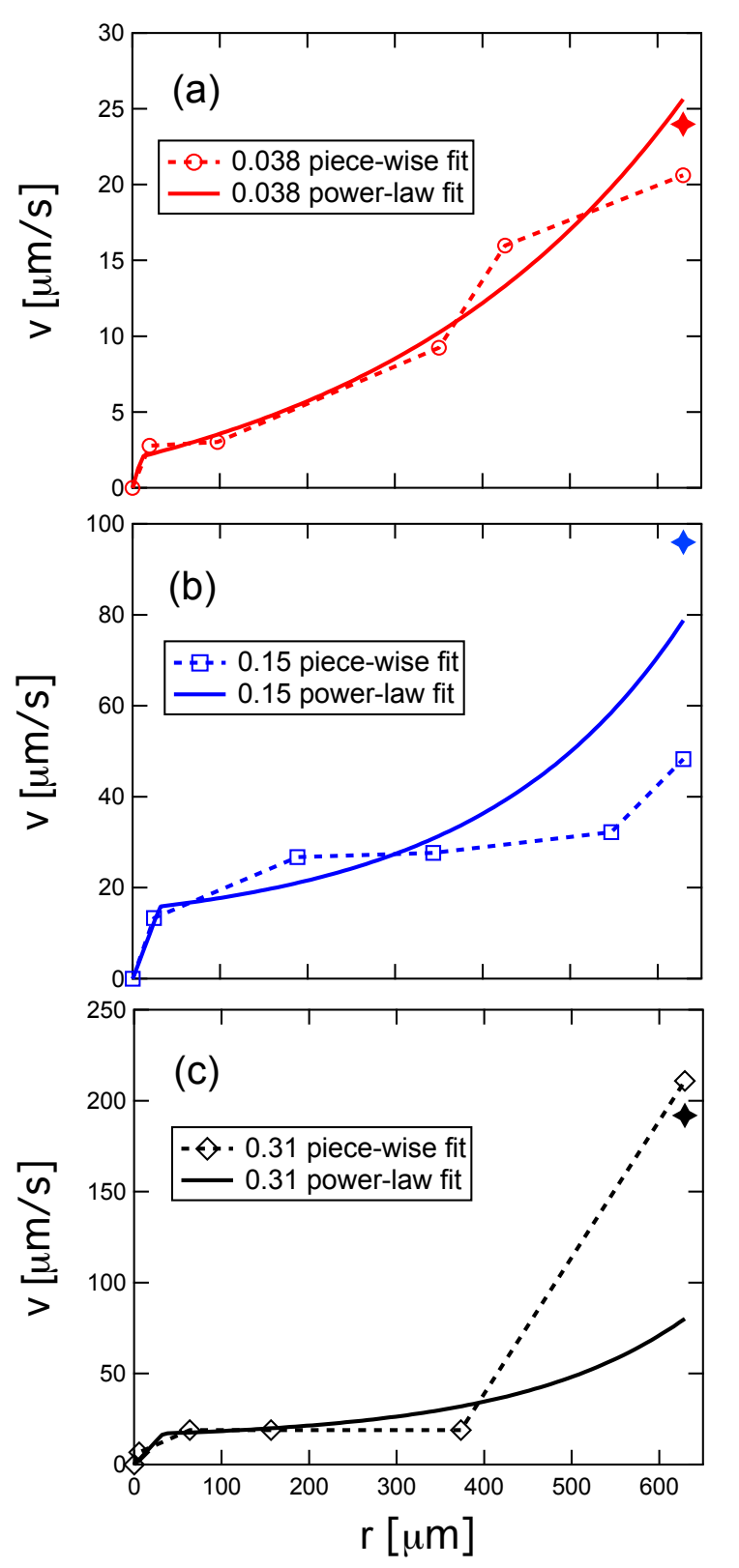

FIG. 8. Velocity profiles in the Gibbsite dispersion as a function of distance $r$ from the Couette-cell outer wall under constant engineered shear rates of (a) 0.038 , (b) 0.15 , and (c) $0.31 \mathrm{~s}^{-1}$ as obtained by fitting the XPCS data with the piece-wise linear model (dashed lines) and power-law model (solid lines). The solid points at $x=629 \mu \mathrm{m}$ indicate the engineered velocity of the inner wall at each shear rate.

shear rates, change with engineered rate, reflecting an evolution in the velocity profile. However, a key similarity among the three profiles is a narrow shear band closest to the outer wall with a high local shear rate, which corresponds to the slip region mentioned above. The thickness of the slip regions are $19.5,24.2$, and $5.6 \mu \mathrm{m}$ under engineered shear rates set by the rheometer of $0.038,0.15$, and $0.31 \mathrm{~s}^{-1}$, respectively, and the shear rate in the region is approximately four times as the engineered shear rate in each case. Moving into the 
gap from this slip region, the velocity profile deviates increasingly from a linear profile as the shear rate increases. The velocity profiles at the engineered shear rates 0.15 and 0.31 $\mathrm{s}^{-1}$, shown in Fig. 8(b) and (c), respectively, display regions in which the local shear rate is nearly zero and hence the velocity is nearly constant, like a plug flow. The width of the low-gradient band extends more than $300 \mu \mathrm{m}$ across the central portion of the gap. In addition, a band with a high shear rate is found between the region of plug-like flow and the inner wall. In contrast, the velocity profile at the engineered shear rate of $0.038 \mathrm{~s}^{-1}$, shown in Figure 8(a), contains a more modest upward curvature beyond the slip region without regions with such pronounced differences in shear rate. The average shear rates based on the fits, obtained by dividing the fitted velocities at the inner wall by the width of the gap, are 0.033 , 0.08 , and $0.34 \mathrm{~s}^{-1}$ for the engineered rates of $0.038,0.15$ and $0.31 \mathrm{~s}^{-1}$, respectively. The discrepancies between the values at the lower two shear rates suggest a narrow slip region also forms at the inner wall. The fact that this slip disappears when the shear bands form is indicative of the competition between shear banding and wall slip ${ }^{11}$.

\section{Power-law model}

The second approach to model the nonlinear velocity profile of the Gibbsite dispersion is based on the fact that the dispersion is a shear-thinning fluid, as illustrated by Fig. 1 . Under certain circumstances, shear thinning fluids are predicted to form a power-law velocity profile under steady shear 52. Specifically, when a fluid under Couette flow displays a power-law stress-strain relation, $\sigma \propto \dot{\gamma}^{m}$, its underlying velocity profile can take on a power-law form,

$$
v(r)=\omega_{0} R_{\text {in }} \frac{r^{1-2 / m}-R_{\mathrm{out}}^{1-2 / m}}{R_{\mathrm{in}}^{1-2 / m}-R_{\mathrm{out}}^{1-2 / m}}
$$

where $r$ is the distance from the outer wall, and $\omega_{0}$ is the angular velocity of the inner wall. As discussed above, irrespective of the modeling, the data from the Gibbsite dispersion shows clear evidence of a region of slip near the outer wall, so in modeling the results with the power-law velocity profile, we also include a slip band with shear rate $\dot{\gamma}_{m}$ and width $\Delta$ near the outer wall. Thus, the full expression for the velocity in the Cartesian coordinates of Fig. 2(b) is

$$
v(x, y)= \begin{cases}\dot{\gamma}_{m}\left[R_{\text {out }}-\sqrt{\left(R_{\text {out }}-x\right)^{2}+y^{2}}\right], & R_{\text {out }}-\sqrt{\left(R_{\text {out }}-x\right)^{2}+y^{2}} \leq \Delta \\ \dot{\gamma}_{m} \Delta+v_{p} \frac{\left[\frac{R_{\text {out }}-\Delta}{\sqrt{\left(R_{\text {out }}-x\right)^{2}+y^{2}}}\right]^{2 / m-1}-1}{\left(\frac{R_{\text {out }}-\Delta}{R_{\text {in }}}\right)^{2 / m-1}-1}, & \Delta<R_{\text {out }}-\sqrt{\left(R_{\text {out }}-x\right)^{2}+y^{2}} \leq H\end{cases}
$$

where $v_{p}$ is the velocity difference across the portion of the gap with the power-law profile. The XPCS correlation functions measured at beam position $x$ for this velocity profile are therefore:

$$
\Delta g_{2}\left(q_{x}, \tau ; x\right)=\frac{e^{-\Gamma_{D} \tau}}{y_{0}^{2}}\left[\int_{0}^{y_{0}} \cos \left[q_{x} \frac{v(x, y) y}{\sqrt{\left(R_{\mathrm{out}}^{2}-x^{2}\right)+y^{2}}} \tau\right] d y\right]^{2} .
$$

Employing the same procedure to that used with the piecewise linear model, we fit Eq. (18) to all eleven $\Delta g_{2}\left(q_{x}, \tau ; x\right)$ across the gap simultaneously for each nominal shear rate with four fitting parameters: the width $\Delta$ and shear rate $\dot{\gamma}$ of the slip region, the shear-thinning exponent $m$, and the velocity increment $v_{p}$. The velocity profiles obtained from these fits are shown in Fig. 8 as solid lines. The profiles obtained with this power-law model show reasonable similarity to those obtained with the piece-wise linear model. In particular, the two models are in good agreement regarding the size and shear rate of the slip region near the outer wall. However, the quality of the fits using the power-law model is inferior to that using the piece-wise linear model, especially at the engineered shear rates of 0.15 and $0.31 \mathrm{~s}^{-1}$. This difference in the quality of the fits is indicated in Fig. 7, where the solid symbols show the discrepancies $\chi$ between the best fits using the powerlaw model and the experimental $\Delta g_{2}\left(q_{x}, \tau ; x\right)$. The power-law model has a larger $\chi$ than the piece-wise linear model with three bands $(n=3)$ despite having one more free parameter. The failure of the power-law model at the higher two shear rates appears to result from the difficulty of the model in capturing the region of plug-like flow in the middle of the cell gap and the dramatic variation in velocity near the inner wall that is produced by the piece-wise linear model. 


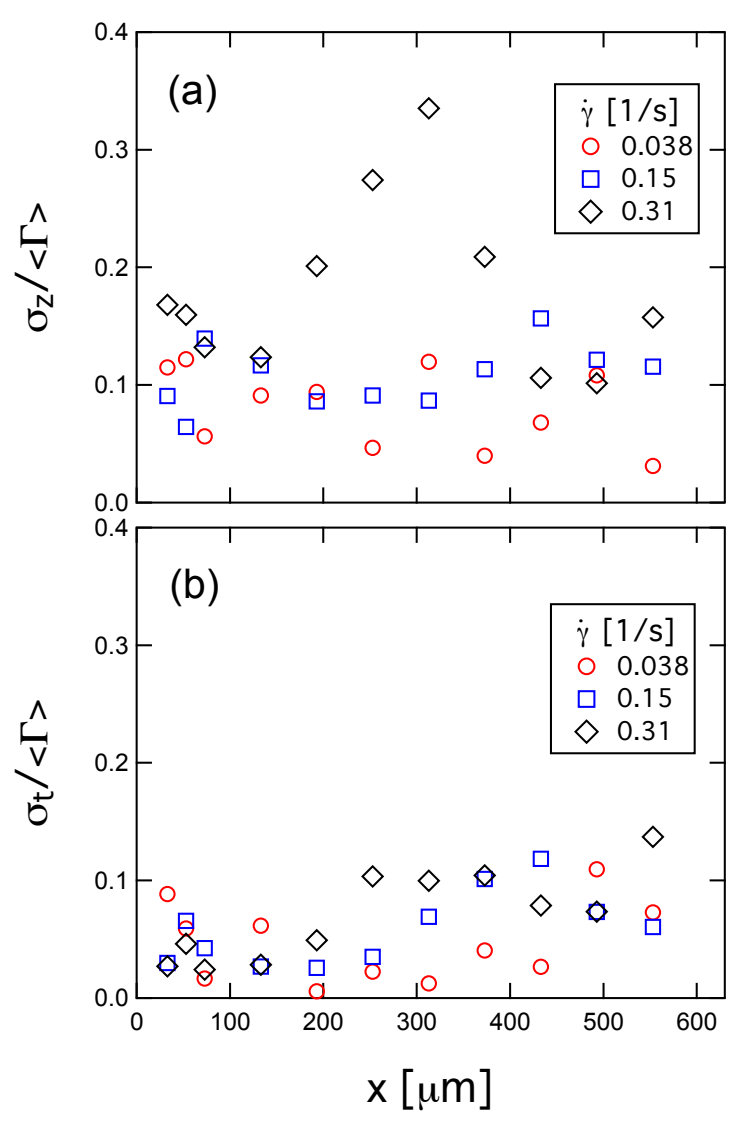

FIG. 9. Standard deviation in the decorrelation rate of $\Delta g_{2}\left(q_{x}, \tau ; x\right)$ normalized by the mean decorrelation rate (a) across five measurements at the same horizontal but different vertical positions in the gap and (b) across five segments of a single measurement as functions of horizontal position. Data is shown for the three engineered shear rates, as indicated in the legend.

\section{Spatial heterogeneity in flow}

As mentioned above, a feature of the flow of the Gibbsite dispersion is spatial heterogeneity in the velocity profile along the vorticity direction, which becomes particularly pronounced at the largest shear rate, $0.31 \mathrm{~s}^{-1}$. Evidence for this heterogeneity comes from comparing XPCS correlation functions obtained at the same horizontal beam position $x$ but at different heights $z$ in the Couette cell. Recall that at each horizontal position a set of five XPCS measurements were performed at beam positions spaced by $60 \mu \mathrm{m}$ along the $z$ (vorticity) direction. To quantify the heterogeneity at different heights, we define the decorrelation rate $\Gamma$ of each normalized correlation function as the inverse of the delay time at which the function decays to $1 / e$ and calculate the standard deviation $\sigma_{z}$ in the decorrelation rate over each set of five measurements. Figure 9(a) displays this standard deviation, normalized by the mean decorrelation rate, as a function horizontal position $x$ across the gap for the three shear rates. The standard deviations at $0.31 \mathrm{~s}^{-1}$ show a pronounced peak near the middle of the Couette cell gap, $x \approx 300 \mu \mathrm{m}$, indicating
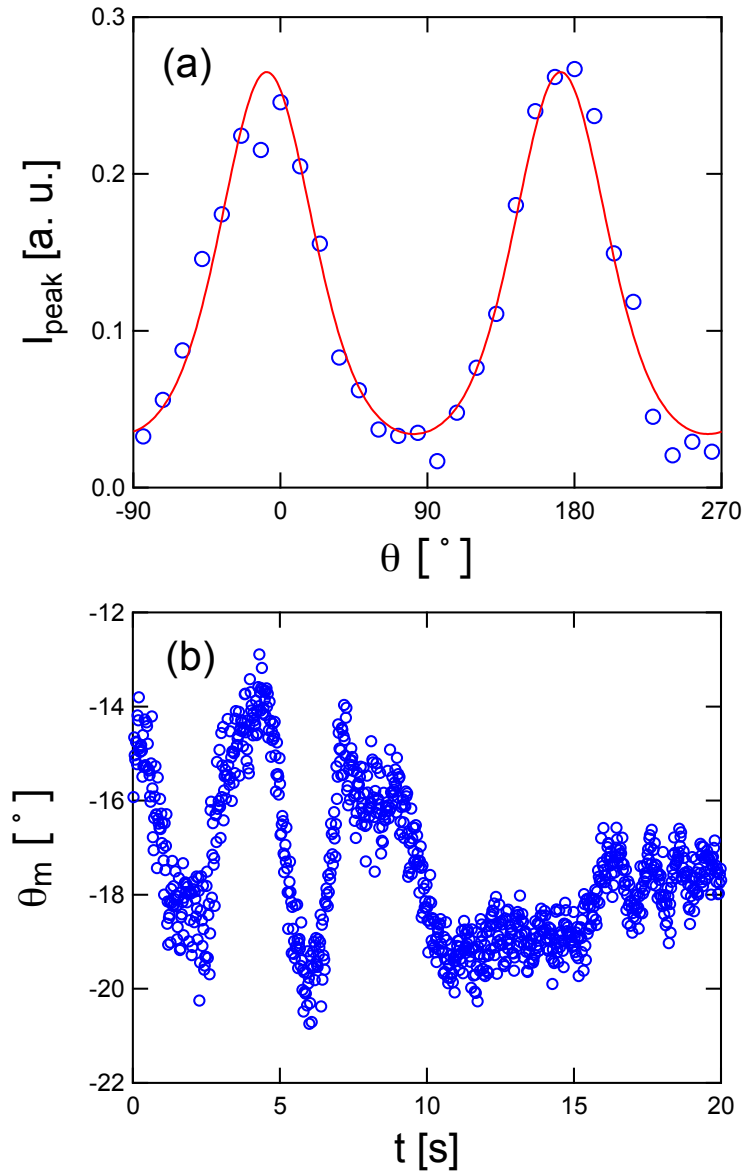

FIG. 10. (a) Scattering intensity at the wave vector of the structure factor peak, $q_{\max }=0.083 \mathrm{~nm}^{-1}$, as a function of azimuthal angle $\theta$ as seen in Fig. 4, where the shear rate is $0.038 \mathrm{~s}^{-1}$ and the beam passes through the side of the Couette cell at $x=55 \mu \mathrm{m}$ from the outer wall. The line shows the result of a fit using Eq. (19). (b) The azimuthal angle of the peak in $I$ as a function of time from a measurement at shear rate $0.31 \mathrm{~s}^{-1}$ and $x=255 \mu \mathrm{m}$ from the outer wall.

large heterogeneity in the velocity profile along the vorticity direction in this region of the gap at the high shear rate. As indicated in Fig. 8(c), this location in the gap coincides with the interface between the region of plug-like flow and the region of high shear rate adjacent to the inner wall. Variations in velocity profile along the vorticity direction have been shown in systems that form shear bands, such as wormlike micelle solutions, as a consequence of instabilities that develop at the interface between bands ${ }^{53-57}$. The heterogeneity at $0.31 \mathrm{~s}^{-1}$ implied by the large $\sigma_{z}$ suggests such instabilities affect the interface between the plug-like flow and high-shear regions. In contrast, $\sigma_{z}$ at shear rates $0.038 \mathrm{~s}^{-1}$ and $0.15 \mathrm{~s}^{-1}$ remains relatively small across the cell gap, indicating no such instabilities form at the lower shear rates. As described above, the $\Delta g_{2}\left(q_{x}, \tau ; x\right)$ in Fig. 5 that are fit to model the velocity profiles are averages over the five measurements at different $z$, hence the strong heterogeneity at $0.31 \mathrm{~s}^{-1}$ has implications for the validity of the modeling and is likely a source of the relatively 
large $\chi$ seen in Fig. 7 at this shear rate. One should therefore interpret the velocity profile, particularly in the inner half of the gap, as an approximate average over the variations in the vorticity direction at this shear rate.

Instabilities in the interface between shear bands can also be expected to fluctuate in time ${ }^{53}$. To obtain a measure of temporal fluctuations in the velocity profile, we divided each 20-second XPCS measurement into five time intervals, each 4 seconds and 4000 frames long, calculated $\Delta g_{2}\left(q_{x}, \tau ; x\right)$ and its decorrelation rate for each segment, and determined the standard deviations $\sigma_{t}$ of these decorrelation rates. As shown in Fig. 5, all $\Delta g_{2}\left(q_{x}, \tau ; x\right)$ fully decay to zero by $\tau=1 \mathrm{~s}$, so the 4-second segments are adequate to determine a decorrelation rate. Figure 9(b) shows the results for $\sigma_{t}$, normalized by the mean decorrelation rates, as a function of position across the gap. The standard deviations show a small apparent increase on moving from the outer to the inner wall, but at all positions the temporal heterogeneity is significantly smaller than the spatial heterogeneity near the middle of the gap at 0.31 $\mathrm{s}^{-1}$, indicating the spatial fluctuations in the velocity profile at this shear rate have a lifetime that is long compared to the 20 -second duration of the measurements.

\section{Microscopic structure under shear}

The strong shear thinning of the Gibbsite dispersion is presumably coupled to the tumbling behavior of the nematic director under steady shear, which also contributes to the nonlinearity of the velocity profile ${ }^{24,25}$. To understand this interdependence between microstructure and rheology better, we characterize the nematic order and its temporal and spatial variations within the gap. Figure 10(a) shows the scattering intensity $I$ seen in Fig. 4 at the wave vector of the peak in the structure factor, $q_{\max }=0.083 \mathrm{~nm}^{-1}$, as a function of azimuthal angle $\theta$ where the shear rate is $0.038 \mathrm{~s}^{-1}$ and the beam passes through the side of the Couette cell at $x=55 \mu \mathrm{m}$ from the outer wall. The azimuthal dependence of the scattered intensity is described by a Maier-Saupe-type orientation distribution function,

$$
I(\theta)=I_{0} \exp \left[\alpha P_{2}\left(\cos \left(\theta-\theta_{m}\right)\right)-1\right],
$$

where $P_{2}\left(\cos \left(\theta-\theta_{m}\right)\right)=\frac{1}{2}\left(3 \cos ^{2}\left(\theta-\theta_{m}\right)-1\right)$ is the secondorder Legendre polynomial, $\alpha$ is a measure of the sharpness of the peak, $I_{0}$ is proportional to the peak intensity, and $\theta_{m}$ is the angle of maximum intensity, which specifies the angle in the vorticity-gradient plane between the nematic director $\hat{n}$ and the gradient direction ${ }^{38,58,59}$. The magnitude of the average orientational order parameter can be determined by

$$
\bar{P}_{2}=\frac{\int_{0}^{\pi} \exp \left[\alpha P_{2}(\theta)\right] P_{2}(\theta) \sin (\theta) d \theta}{\int_{0}^{\pi} \exp \left[\alpha P_{2}(\theta)\right] \sin (\theta) d \theta} .
$$

Figure 11 shows the time-average values $\left\langle\theta_{m}\right\rangle$ and $\left\langle\bar{P}_{2}\right\rangle$ along with those of the wave-vector position of the peak $\left\langle q_{\max }\right\rangle$ and the average intensity at the peak $\left\langle I_{\text {peak }}\right\rangle$ as functions of position in the gap. The error bars indicate the standard deviations over the five measurements at each horizontal beam position. These parameters characterizing the nematic structure show a dependence on the shear rate that correlates with the variation in the velocity profile. At the two lower shear rates, $\left\langle\theta_{m}\right\rangle$ is close to zero, and $\left\langle\bar{P}_{2}\right\rangle$ is roughly constant except in and near the slip region at the outer wall where $\left\langle\theta_{m}\right\rangle$ shows large fluctuations with position along the vorticity direction and the order parameter is suppressed. In contrast, at $0.31 \mathrm{~s}^{-1}$ $\left\langle\theta_{m}\right\rangle$ deviates sharply from zero at $x \approx 300 \mu \mathrm{m}$, the position of the interface between the region of plug-like flow and the high-shear-rate band, indicating the director orientation is affected by the instability in the interface discussed above. Further, $\left\langle\bar{P}_{2}\right\rangle$ increases discontinuously near $x \approx 300 \mu \mathrm{m}$, indicating the degree of nematic order is enhanced and has high stability in the high-shear-rate band. Meanwhile, $\left\langle q_{\max }\right\rangle$ assumes a roughly constant value, $q_{\max } \approx 0.83 \mathrm{~nm}^{-1}$, across the gap at the lower shear rates but is notably larger and positiondependent at $0.31 \mathrm{~s}^{-1}$. We interpret the reduction in $q_{\max }$ with a shear-flow induced tilt of the director in the flow-gradient plane by an amount known as the Leslie angle ${ }^{60}$. This tilt is the smallest and hence $q_{\max }$ is largest at $0.31 \mathrm{~s}^{-1}$ in the region of plug-like flow due to the wall anchoring and minimal shear-induced tilt. We interpret the smaller $q_{\max }$ and hence larger tilt at the lower shear rates as a result of reduced influence of wall anchoring due to the wider slip region at the outer wall at those shear rates.

Figure 10(b) shows the temporal variation of $\theta_{m}$ during one measurement, indicating fluctuations in the director during the steady shear. The stability of the structure can be characterized by the temporal autocorrelation of $\theta_{m}$, defined as

$$
\operatorname{Corr} \theta_{m}(\tau)=\left\langle\cos \left[\theta_{m}(t+\tau)-\theta_{m}(t)\right]\right\rangle_{t},
$$

where the brackets signify averaging over all frames in one measurement. Figure 12 shows $\operatorname{Corr} \theta_{m}$ as a function of delay time at different positions in the gap at the three shear rates. The five curves in each panel correspond to the five measurements at different heights at each horizontal position. Overall, the correlation function decays very little over the time of a measurement and contains no periodic structure. At the smallest nominal shear rate, the correlation curves decay more slowly, indicating greater stability. At an intermediate nominal shear rate, the correlation curves show large decay at positions closer to the outer wall, and this behavior expands further into the middle of the gap at the largest shear rate. Further, at the highest shear rate, the correlation curves show a fast decay and large variation among different measurements in the half of the gap closer to the outer wall, while the other half, which coincides approximately with the high-shear-rate band, has a much more stable orientation, consistent with the behavior seen in Fig. 11.

\section{DISCUSSION}

We have shown how one can extract a complex velocity profile under Couette flow, as illustrated in Fig. 8, from XPCS measurements by scanning the gap with a beam that is directed along the tangent of the Couette cell, so that the 

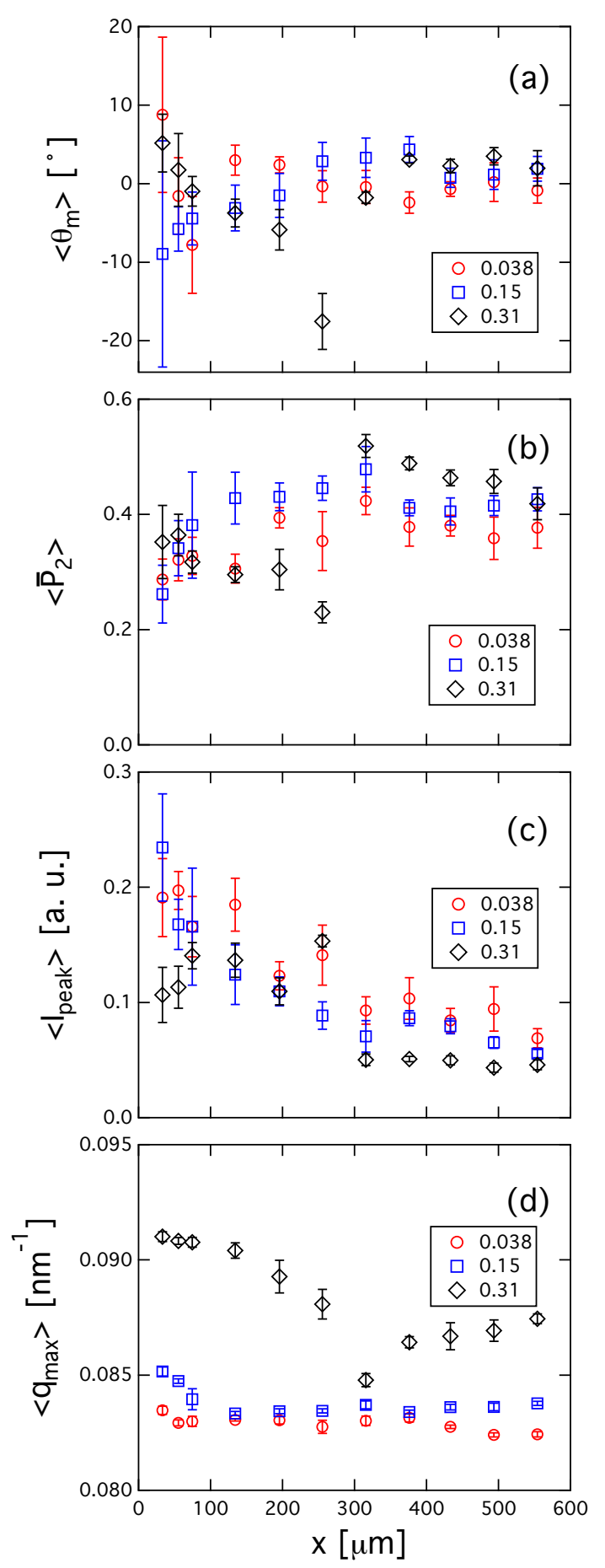

FIG. 11. Time average (a) angle between the nematic director and the gradient direction, (b) order parameter, (c) amplitude of the intensity at the structure factor peak, and (d) magnitude of wave vector of the peak of the scattering pattern as functions of horizontal position in the gap at the engineered shear rates indicated in the legend. Each point is the mean across five measurements at the same lateral position and at different vertical positions, and the error bars represent standard deviations across the five measurements.

scattering plane coincides approximately with the gradient- vorticity plane. We can draw three main conclusions from Fig. 8 regarding the non-Newtonian flow behavior of the nematic Gibbsite suspension that further illustrate the strengths and weaknesses of this XPCS-based approach. First, a narrow slip region at the outer wall forms at all shear rates. The small beam size and small beam divergence ensure that we can resolve this region well. This characterization could potentially be further improved beyond what we demonstrate here by employing an even narrower beam $\left(<2 \mu \mathrm{m}\right.$ is achievable $\left.{ }^{61}\right)$ and more tightly spaced measurements across the gap. In doing so, the spatial resolution achieved with the approach could reach micrometer scale, which is superior to established techniques for obtaining velocity profiles, such as USV, NMR, and heterodyne DLS. Moreover, the fact that the measurements near the outer wall depend only on the flow behavior in that region and are not convolved with the flow behavior in other regions makes the interpretation of the slip region unambiguous.

Second, some slip appears to be present at the inner wall at the two lower shear rates, but at the highest shear rate, the slip in this region appears negligible. The evidence for this slip at the inner wall, which is based on a comparison of the extrapolated flow velocity and the engineered velocity at the wall, is only indirect, however. Direct characterization of the flow at the inner wall would have required positioning the beam precisely at the wall, and even then the results of the measurement would depend not only on the flow behavior in the nearwall region but also on the flow profile throughout the gap. Indeed, as described above, this interdependence is a complicating factor in the analysis of measurements away from the outer wall that is a drawback of this approach.

Third, at the highest shear rate, we can identify two bands in addition to the slip region at the outer wall, one of which displays a plug-like flow with negligible shear rate and one with a high shear rate near $\dot{\gamma}_{h i g h}=1 \mathrm{~s}^{-1}$, or approximately three times the engineered rate. The results further suggest a sharp interface between these bands, but this conclusion is complicated by the observed spatial heterogeneity of the velocity profile along the vorticity direction, which is most pronounced near the interface. This ability to resolve spatially heterogeneity in the velocity profile along the vorticity direction is another strength of the approach. Further, the XPCS results together with the SAXS results suggest that the suspension at the highest shear rate is strongly inhomogeneous both in the dynamic response and structure, as also observed for liquid crystalline graphene oxide ${ }^{29}$. Specifically, the fluctuations in the orientation of the nematic director, as characterized by the decay of $\operatorname{Corr} \theta_{m}(\tau)$, are most pronounced in the region of plug-like flow near the interface with the highshear band (see the third column of Fig. 12 at $x=255$ and $315 \mu \mathrm{m})$. Inside the high-shear band $(x \gtrsim 350 \mu \mathrm{m})$ these fluctuations become much smaller, while also the spatial heterogeneity in the flow profile along the vorticity direction disappears. These changes are further accompanied by changes in the static structure, as seen in Fig. 11, where the average director orientation $\left\langle\theta_{\max }\right\rangle$, order parameter $\left\langle\bar{P}_{2}\right\rangle$, and intensity $\left\langle I_{\text {peak }}\right\rangle$ are plotted versus position in the gap. Again, for $\dot{\gamma}=0.31 \mathrm{~s}^{-1}$ an abrupt change is observed between $x=255$ and $315 \mu \mathrm{m}$. Thus, the formation of bands of different local 

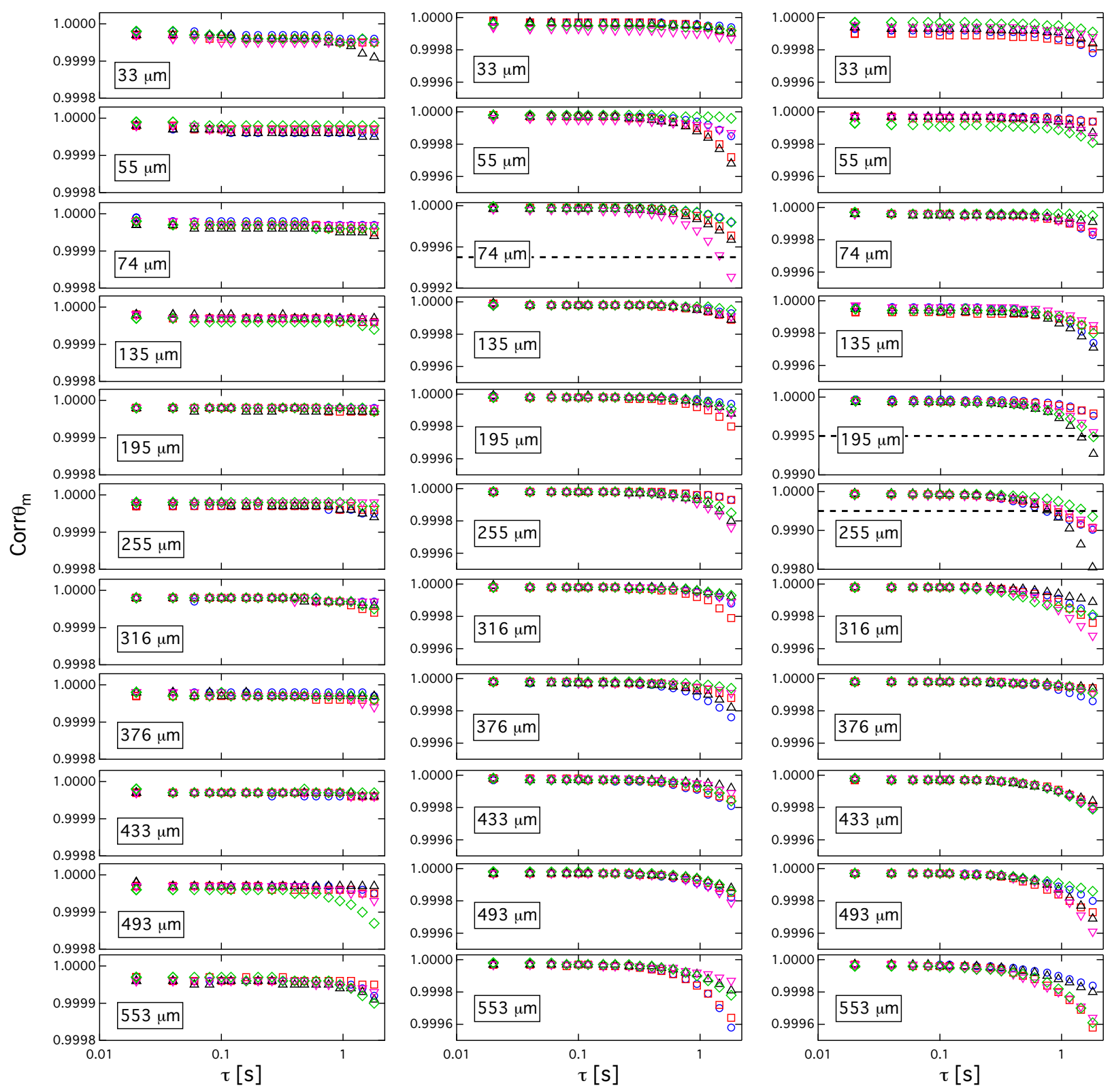

FIG. 12. Temporal autocorrelation of $\theta_{m}$ as a function of delay time at different positions in the gap as labeled in each panel under engineered shear rates 0.038 (left column), 0.15 (middle column), and $0.31 \mathrm{~s}^{-1}$ (right column). Each panel includes five sets of data corresponding to measurements at the same lateral position but at different heights. Dashed horizontal lines at a value 0.9995 are included in some panels to aid comparison.

shear rates coincides with the formation of bands of differing structure, which we can probe throughout the gap with a high spatial resolution. We can thus conclude the flow is not stable around the interface, similar to what has been observed previously in shear-thinning dispersions of Xanthan ${ }^{52}$. Our observation of shear-band formation by the dispersion of nematic platelets at high shear rates confirms the prediction that the interplay between wall anchoring and tumbling motion can induce a flow instability ${ }^{24,25}$, for which only structural evidence in Poisseuille flow $^{36}$ and Large Amplitude Oscillatory Shear was previously available ${ }^{60}$. For the lower engineered shear rates, these averaged values change smoothly with no abrupt jumps, which is consistent with the relatively smooth nonlinear velocity profiles at these shear rates. This ability to characterize the velocity profile and microstructure simultaneously is another strength of the experimental approach.

\section{CONCLUSION}

In conclusion, the combination of spatially resolved structural and dynamic information provided in one go by the rheo- 
XPCS technique demonstrated here has enabled us to characterize several key features of the nonlinear flow behavior of nematic platelet suspensions and its coupling to the nematic structure including (i) the presence of a slip region near the stationary wall, (ii) the formation of a band of plug-like flow with increasing shear rate, and (iii) an instability in the interface between this band and an adjacent high-shear band. One can imagine applying this technique to understand the flow behavior of a host of other non-Newtonian fluids, particularly those with a nanoscale structure that couples to the flow and is amenable to interrogation with small-angle $\mathrm{x}$-ray scattering. Looking to the future, one can further anticipate opportunities to expand this application of XPCS by leveraging enhanced capabilities that ongoing and scheduled upgrades to synchrotrons will afford. Specifically, the increased coherent x-ray flux that the APS and other facilities are projected to achieve in the coming years should enable XPCS measurements using smaller beams and probing more weakly scattering samples than possible today. They should also introduce the possibility of conducting XPCS measurements with higher-energy $\mathrm{x}$-rays (to at least $\approx 25 \mathrm{keV}$ ) whose penetration depth will facilitate measurements on samples under flow in environments that are not feasible today. The principles introduced here in this rheo-XPCS study of Gibbsite suspensions should provide a framework for this work.

\section{SUPPLEMENTARY MATERIAL}

Correlation functions $g_{2}$ measured with both silica and Gibbsite colloid dispersions at various shear rates and all eleven beam positions in the gap of Couette cell are shown in Supplementary Material.

\section{ACKNOWLEDGMENTS}

Funding was provided by the NSF (CBET-1804721). The research used resources of the Advanced Photon Source and the Center for Nanoscale Materials, U.S. Department of Energy (DOE) Office of Science User Facilities operated for the DOE Office of Science by Argonne National Laboratory under Contract No. DE-AC02-06CH11357.

\section{DATA AVAILABILITY STATEMENT}

The data that support the findings of this study are available from the corresponding author upon reasonable request.

${ }^{1}$ J. J. Wang, D. Yavich, and L. G. Leal, "Time-resolved velocity gradient and optical anisotropy in linear flow by photon correlation spectroscopy," Physics of Fluids 6, 3519-3534 (1994).

${ }^{2}$ J.-B. Salmon, S. Manneville, A. Colin, and B. Pouligny, "An optical fiber based interferometer to measure velocity profiles in sheared complex fluids," The European Physical Journal Applied Physics 22, 143-154 (2003). ${ }^{3}$ J. K. G. Dhont, K. Kang, H. Kriegs, O. Danko, J. Marakis, and D. Vlassopoulos, "Nonuniform flow in soft glasses of colloidal rods," Physical Review Fluids 2, 043301 (2017).
${ }^{4}$ S. Jaradat, M. Harvey, and T. A. Waigh, "Shear-banding in polyacrylamide solutions revealed via optical coherence tomography velocimetry," Soft Matter 8, 11677-11686 (2012).

${ }^{5} \mathrm{Y}$. T. Hu and A. Lips, "Kinetics and mechanism of shear banding in an entangled micellar solution," Journal of Rheology 49, 1001-1027 (2005).

${ }^{6}$ Y. Wei, M. J. Solomon, and R. G. Larson, "Time-dependent shear rate inhomogeneities and shear bands in a thixotropic yield-stress fluid under transient shear," Soft Matter 15, 7956-7967 (2019).

${ }^{7}$ P. Callaghan, "Rheo-NMR and velocity imaging," Current Opinion in Colloid \& Interface Science 11, 13-18 (2006).

${ }^{8}$ P. T. Callaghan, "Rheo NMR and shear banding," Rheologica Acta 47, 243 255 (2008).

${ }^{9}$ R. N. Al-kaby, J. S. Jayaratne, T. I. Brox, S. L. Codd, J. D. Seymour, and J. R. Brown, "Rheo-nmr of transient and steady state shear banding under shear startup," Journal of Rheology 62, 1125-1134 (2018).

${ }^{10}$ S. Manneville, L. Bècu, P. Grondin, and A. Colin, "High-frequency ultrasonic imaging: A spatio-temporal approach of rheology," Colloids and Surfaces A: Physicochemical and Engineering Aspects 270-271, 195 - 204 (2005).

${ }^{11}$ M. P. Lettinga and S. Manneville, "Competition between shear banding and wall slip in wormlike micelles," Phys. Rev. Lett. 103, 248302 (2009).

${ }^{12}$ T. Gallot, C. Perge, V. Grenard, M.-A. Fardin, N. Taberlet, and S. Manneville, "Ultrafast ultrasonic imaging coupled to rheometry: Principle and illustration," Review of Scientific Instruments 84, 045107 (2013).

${ }^{13}$ C. Poelma, "Ultrasound Imaging Velocimetry: a review," Experiments in Fluids 58, 3 (2016).

${ }^{14}$ R. L. Leheny, M. C. Rogers, K. Chen, S. Narayanan, and J. L. Harden, "Rheo-xpcs," Current Opinion in Colloid \& Interface Science 20, 261 271 (2015).

${ }^{15} \mathrm{~S}$. Busch, T. Jensen, Y. Chushkin, and A. Fluerasu, "Dynamics in shear flow studied by x-ray photon correlation spectroscopy," Eur. Phys. J. E 26, 55-62 (2008).

${ }^{16}$ A. Fluerasu, A. Moussaï, P. Falus, H. Gleyzolle, and A. Madsen, "Xray photon correlation spectroscopy under flow," J. Synchrotron Radiat. 15, 378-384 (2008).

${ }^{17}$ R. Urbani, F. Westermeier, B. Banusch, M. Sprung, and T. Pfohl, "Brownian and advective dynamics in microflow studied by coherent X-ray scattering experiments," Journal of Synchrotron Radiation 23, 1401-1408 (2016).

${ }^{18}$ J. R. M. Lhermitte, M. C. Rogers, S. Manet, and M. Sutton, "Velocity measurement by coherent $\mathrm{X}$-ray heterodyning," Review of Scientific Instruments 88, 015112 (2017).

${ }^{19}$ W. R. Burghardt, M. Sikorski, A. R. Sandy, and S. Narayanan, "X-ray photon correlation spectroscopy during homogenous shear flow," Phys. Rev. E 85, 021402 (2012).

${ }^{20} \mathrm{~F}$. Westermeier, D. Pennicard, H. Hirsemann, U. H. Wagner, C. Rau, H. Graafsma, P. Schall, M. Paul Lettinga, and B. Struth, "Connecting structure, dynamics and viscosity in sheared soft colloidal liquids: a medley of anisotropic fluctuations," Soft Matter 12, 171-180 (2016).

${ }^{21}$ Y. Chen, S. A. Rogers, S. Narayanan, J. L. Harden, and R. L. Leheny, "Microscopic dynamics of stress relaxation in a nanocolloidal soft glass," Phys. Rev. Materials 4, 035602 (2020).

${ }^{22} \mathrm{~S}$. M. Jogun and C. F. Zukoski, "Rheology and microstructure of dense suspensions of plate-shaped colloidal particles," Journal of Rheology 43, 847-871 (1999).

${ }^{23}$ R. G. Larson, "Arrested tumbling in shearing flows of liquid crystal polymers," Macromolecules 23, 3983-3992 (1990).

${ }^{24}$ D. Grecov and A. D. Rey, "Transient rheology of discotic mesophases," Rheologica Acta 42, 590-604 (2003).

${ }^{25}$ D. Grecov and A. D. Rey, "Theoretical and Computational Rheology for Discotic Nematic Liquid Crystals," Molecular Crystals and Liquid Crystals 391, 57-94 (2003).

${ }^{26}$ C. Lang, J. Kohlbrecher, L. Porcar, A. Radulescu, K. Sellinghoff, J. K. G. Dhont, and M. P. Lettinga, "Microstructural Understanding of the Lengthand Stiffness-Dependent Shear Thinning in Semidilute Colloidal Rods," Macromolecules 52, 9604-9612 (2019).

${ }^{27}$ M. P. Lettinga, Z. Dogic, H. Wang, and J. Vermant, "Flow behavior of colloidal rodlike viruses in the nematic phase," Langmuir 21, 8048-8057 (2005).

${ }^{28}$ A. Brown and A. Rennie, "Images of shear-induced phase separation in a dispersion of hard nanoscale discs," Chemical Engineering Science 56, 
2999-3004 (2001).

${ }^{29}$ J. K. Wychowaniec, M. Iliut, B. Borek, C. Muryn, O. O. Mykhaylyk, S. Edmondson, and A. Vijayaraghavan, "Elastic flow instabilities and macroscopic textures in graphene oxide lyotropic liquid crystals," npj 2D Materials and Applications 5, 11 (2021).

${ }^{30}$ E. P. Choate, M. G. Forest, and L. Ju, "Effects of strong anchoring on the dynamic moduli of heterogeneous nematic polymers II: Oblique anchoring angles," Rheologica Acta 49, 335-347 (2010).

${ }^{31}$ S. Heidenreich, S. Hess, and S. H. L. Klapp, "Shear-induced dynamic polarization and mesoscopic structure in suspensions of polar nanorods," Phys. Rev. Lett. 102, 028301 (2009).

${ }^{32}$ V. M. O. Batista, M. L. Blow, and M. M. Telo da Gama, "The effect of anchoring on the nematic flow in channels," Soft Matter 11, 4674-4685 (2015).

${ }^{33}$ L. M. Dykes, J. M. Torkelson, W. R. Burghardt, and R. Krishnamoorti, "Shear-induced orientation in polymer/clay dispersions via in situ X-ray scattering," Polymer 51, 4916-4927 (2010).

${ }^{34}$ L. Harnau and S. Dietrich, "Fluids of platelike particles near a hard wall," Physical Review E 65, 021505 (2002).

${ }^{35}$ A. Verhoeff, R. Brand, and H. Lekkerkerker, "Tuning the birefringence of the nematic phase in suspensions of colloidal gibbsite platelets," Molecular Physics 109, 1363-1371 (2011).

${ }^{36}$ S. Barè, J. K. Cockcroft, S. L. Colston, A. C. Jupe, and A. R. Rennie, "Xray study of the orientational order of a concentrated dispersion of kaolinite under flow," Journal of Applied Crystallography 34, 573-579 (2001).

${ }^{37}$ M. P. Lettinga, P. Holmqvist, P. Ballesta, S. Rogers, D. Kleshchanok, and B. Struth, "Nonlinear behavior of nematic platelet dispersions in shear flow," Phys. Rev. Lett. 109, 246001 (2012).

${ }^{38}$ O. Korculanin, D. Hermida-Merino, H. Hirsemann, B. Struth, S. A. Rogers, and M. P. Lettinga, "Anomalous structural response of nematic colloidal platelets subjected to large amplitude stress oscillations," Physics of Fluids 29, 023102 (2017).

${ }^{39}$ A. Bharati, S. D. Hudson, and K. M. Weigandt, "Poiseuille and extensional flow small-angle scattering for developing structure - rheology relationships in soft matter systems," Current Opinion in Colloid \& Interface Science 42, 137-146 (2019).

${ }^{40}$ K. Nygård, J. Buitenhuis, M. Kagias, K. Jefimovs, F. Zontone, and Y. Chushkin, "Anisotropic de Gennes Narrowing in Confined Fluids," Phys. Rev. Lett. 116, 167801 (2016).

${ }^{41}$ J. Gapinski, A. Patkowski, A. J. Banchio, J. Buitenhuis, P. Holmqvist, M. P. Lettinga, G. Meier, and G. Nägele, "Structure and short-time dynamics in suspensions of charged silica spheres in the entire fluid regime," J. Chem. Phys. 130, 084503 (2009).

${ }^{42}$ D. Kleshchanok, J.-M. Meijer, A. V. Petukhov, G. Portale, and H. N. W. Lekkerkerker, "Attractive glass formation in aqueous mixtures of colloidal gibbsite platelets and silica spheres," Soft Matter 7, 2832-2840 (2011).

${ }^{43}$ S. Jabbari-Farouji, J.-J. Weis, P. Davidson, P. Levitz, and E. Trizac, "On phase behavior and dynamical signatures of charged colloidal platelets," Scientific Reports 3, 3559 (2013).
${ }^{44}$ D. van der Beek and H. N. W. Lekkerkerker, "Liquid crystal phases of charged colloidal platelets," Langmuir 20, 8582-8586 (2004).

${ }^{45}$ S. Jogun and C. F. Zukoski, "Rheology of dense suspensions of platelike particles," Journal of Rheology 40, 1211-1232 (1996).

${ }^{46}$ D. Pennicard, S. Lange, S. Smoljanin, J. Becker, H. Hirsemann, M. Epple and H. Graafsma, "Development of LAMBDA: Large area medipix-based detector array," J. Instrum. 6, C11009 (2011).

${ }^{47}$ F. Khan, S. Narayanan, R. Sersted, N. Schwarz, and A. Sandy, "Distributed X-ray photon correlation spectroscopy data reduction using Hadoop MapReduce," J. Synchrotron Radiat. 25, 1135 (2018).

${ }^{48}$ K. Schätzel, "Correlation techniques in dynamic light scattering," Appl. Phys. B 42, 193-213 (1987).

${ }^{49}$ G. G. Fuller, J. M. Rallison, R. L. Schmidt, and L. G. Leal, "The measurement of velocity gradients in laminar flow by homodyne light-scattering spectroscopy,” J. Fluid Mech. 100, 555-575 (1980).

${ }^{50} \mathrm{M}$. Cloitre and R. T. Bonnecaze, "A review on wall slip in high solid dispersions,” Rheologica Acta 56, 283-305 (2017).

${ }^{51}$ A. Y. Malkin and S. Patlazhan, "Wall slip for complex liquids - phenomenon and its causes," Advances in Colloid and Interface Science 257, 42-57 (2018).

${ }^{52}$ H. Tang, T. Kochetkova, H. Kriegs, J. K. G. Dhont, and M. P. Lettinga, "Shear-banding in entangled xanthan solutions: tunable transition from sharp to broad shear-band interfaces," Soft Matter 14, 826-836 (2018).

${ }^{53}$ S. Lerouge, M. Argentina, and J. P. Decruppe, "Interface instability in shear-banding flow," Phys. Rev. Lett. 96, 088301 (2006).

${ }^{54}$ M. A. Fardin, B. Lasne, O. Cardoso, G. Grégoire, M. Argentina, J. P. Decruppe, and S. Lerouge, "Taylor-like Vortices in Shear-Banding Flow of Giant Micelles,” Physical Review Letters 103, 028302 (2009).

${ }^{55}$ M. A. Fardin, D. Lopez, J. Croso, G. Grégoire, O. Cardoso, G. H. McKinley, and S. Lerouge, "Elastic Turbulence in Shear Banding Wormlike Micelles," Physical Review Letters 104, 178303 (2010).

${ }^{56}$ M. A. Fardin and S. Lerouge, "Instabilities in wormlike micelle systems: From shear-banding to elastic turbulence," The European Physical Journal E 35, 91 (2012).

${ }^{57} \mathrm{H}$. Mohammadigoushki and S. J. Muller, "Inertio-elastic instability in taylor-couette flow of a model wormlike micellar system," Journal of Rheology 61, 683-696 (2017).

${ }^{58}$ S. J. Picken, J. Aerts, R. Visser, and M. G. Northolt, "Structure and rheology of aramid solutions: X-ray scattering measurements," Macromolecules 23, 3849-3854 (1990).

${ }^{59}$ B. Lonetti, J. Kohlbrecher, L. Willner, J. K. G. Dhont, and M. P. Lettinga, "Dynamic response of block copolymer wormlike micelles to shear flow," Journal of Physics: Condensed Matter 20, 404207 (2008).

${ }^{60}$ O. Korculanin, F. Westermeier, H. Hirsemann, B. Struth, S. A. Rogers, and M. P. Lettinga, "Revealing microstructural inhomogeneities in sheared nematic dispersions of colloidal platelets," (2021).

${ }^{61}$ E. M. Dufresne, S. Narayanan, R. Reininger, A. R. Sandy, and L. Lurio, "Focusing a round coherent beam by spatial filtering the horizontal source," Journal of Synchrotron Radiation 27, 1528-1538 (2020). 